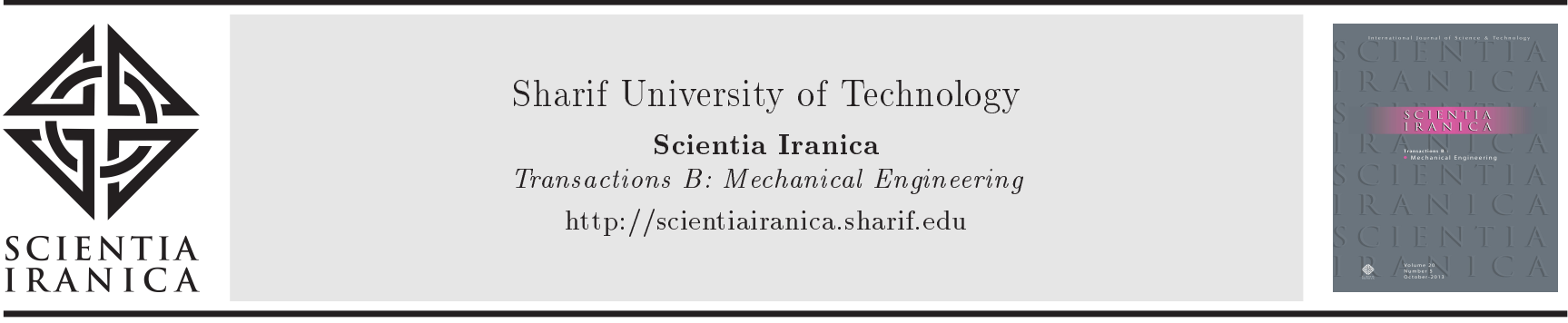

\title{
Free vibration response of functionally graded carbon nanotube double curved shells and panels with piezoelectric layers in a thermal environment
}

\author{
S. Khorshidi ${ }^{a, *}$, S. Saber-Samandari ${ }^{b}$, and M. Salehi ${ }^{a}$ \\ a. Department of Mechanical Engineering, Amirkabir University of Technology, Tehran, Iran. \\ b. New Technologies Research Center, Amirkabir University of Technology, Tehran, Iran. \\ Received 2 September 2018; received in revised form 27 March 2019; accepted 28 May 2019
}

\author{
KEYWORDS \\ Vibration; \\ Piezoelectric layer; \\ CNT; \\ Thermal analysis; \\ Reissner-Mindlin \\ theory.
}

\begin{abstract}
This paper focuses on free vibration of double-curved shells and panels with piezoelectric layers in a thermal environment. Vibration characteristics of elliptical, spherical, cycloidal, and toro circular shells of revolution structures were studied in detail. These structures are made of a Carbon Nanotube (CNT) core and piezoelectric layers on the upper and lower surfaces. It was assumed here that temperature varied linearly in the thickness direction. Reissner-Mindlin and the first-order shear deformation theories were implemented to derive the governing equations of the considered structures. The distribution of nanotubes was assumed linear along the thickness direction. To solve the equation, the Generalized Differential Quadrature (GDQ) method was implemented to investigate the dynamic behavior of the structures. Finally, the effects of the boundary conditions, the thickness of piezoelectric layers, the functional distribution of CNTs, thermal environment, and two kinds of circuit (open circuit and closed circuit) were analyzed. Eigenvalue system was solved to obtain natural frequencies. Results showed that the obtained fundamental frequency of the closed circuit was smaller than that of the open circuit. Another interesting result was that the natural frequency was reduced at higher temperatures.
\end{abstract}

(C) 2020 Sharif University of Technology. All rights reserved.

\section{Introduction}

Shells are three-dimensional structures that are characterized by unique mechanical properties and constrained by two curved surfaces. Generally, these structures are divided into three groups such as thick, moderately thick, and thin shells [1]. Because of their excellent and unique mechanical characteristics, they are implemented in different applications including

\footnotetext{
*. Corresponding author:

E-mail address: shahruz@aut.ac.ir (S. Khorshidi)
}

doi: $10.24200 /$ sci. 2019.51698 .2319 submarines, storages, tanks, automobiles, airplanes, etc. [2]. One of the investigation aspects of shells is analysis of their vibration characteristics. Therefore, considering their extensive applications to different mechanical and civil structures, it is vital to develop an accurate and reliable dynamical model for shells. Shells can not only be assumed as the 3D body, but also behave as 2D structures. In terms of their 3D applications, the governing equations are derived using the $3 \mathrm{D}$ elasticity theory and for $2 \mathrm{D}$ applications, the shell theory is implemented. In the shell theory $(2 \mathrm{D}$ model), the mid-surface of a structure is considered to be a reference plane, and the system of equations is derived based on the assumed reference plane. The 
present study used this theory to extract the governing equations. In this study, piezoelectric layers are embedded in the upper and lower surfaces. As a result, it is necessary to adjust piezoelectric applications for industries. In practice, they can be used for power generation in automobiles. When an automobile's tires are rotating on the roads, a certain amount of energy is wasted. This kind of energy supplies the required power to automobile electronic devices. It should be mentioned that piezoelectric materials can be used to generate electrical energy when subjected to mechanical strain [3]. Another application is the tire pressure sensor, which is placed inside a wheel; by measuring the amount of force imported to the piezoelectric disc, the sensor can be an accurate indicator for regulating the tire pressure. As implied earlier, the characteristic of the piezoelectric material, which converts electrical energy into mechanical energy and vice versa, provides the potential to reap its benefit [3]. In recent years, there has been a great effort for modeling and analyzing the static and dynamic properties of the moderately thick shells. For example, Tornabene [4] probed the free vibration of anisotropic double shells using twodimensional differential quadrature method. They implemented the first-order shear deformation theory and investigated the vibration behavior of the moderately thick structures. Viola et al. [5] conducted static analysis of double-curved shells and panels with higher order shear deformation theory. To this end, they discretized the governing equations of the structure by using the Generalized Differential Quadrature (GDQ) method. Shooshtari and Razavi [6] used single-mode Galerkin method to study large amplitude vibration of magneto-electro-elastic curved panels. In this research, they implemented Donnell shell theory and Gauss's laws for electrostatics and magnetostatics to derive the governing equations. Finally, they examined the first mode of vibration of this structure. Pang et al. [7] employed a semi-analytical method to analyze the free vibration of joined spherical-cylindrical-spherical shells. They utilized the Flugge thin shell theory to extract governing equations. To approximate the displacement function, unified Jacobi polynomials and Fourier series were used. Finally, the results were compared with those which were obtained from the Finite Element Method (FEM). Rout et al. [8] conducted the free vibration analysis of graphene-reinforced shells and panels in a thermal environment. In this research, the governing equations of four types of double-curved shells were solved with FEM. Moreover, materials were defined based on the Halpin-Tsai approach to showing their dependence on temperature. Finally, the results were contrasted by other studies to confirm their credits. Pang et al. [9] analyzed free vibration of the double-curved shell of revolution with a semi-analytical method. The displacements along the revolution axis were approximated with the Jacobi polynomials, and the Fourier series were used to indicate the movement along the circumferential direction. The Rayleigh-Ritz method was implemented to obtain natural frequencies. Awrejcewicz et al. [10] studied linear and nonlinear free vibrations of laminated functionally graded shallow shells. They assumed that these structures were made of Functionally Graded Material (FGM) based on power law and varied along the thickness direction. The first shear deformation theory was applied to define the displacement field. To solve equations, the Ritz and the $R$-functions method were utilized simultaneously. Fang et al. [11] applied GoldenveizerNovozhilov shell theory, thin plate theory, and electroelastic surface theory to investigate nano-sized piezoelectric double-shell structures. In this research, the surface energy effect was considered, and the governing equation was solved by using the Rayleigh-Ritz method.

Zhou et al. [12] applied an accurate approach to analyzing the free vibration of piezoelectric fiberreinforced composite cylindrical shells in a thermal environment. They assumed that composite materials were made in two ways: first, each ply had a regular distribution and, second, reinforcements were graded functionally through the thickness direction. The governing equations were extracted by using the Hamilton method and, for solving them, an analytical approach was applied. Mallek et al. [13] analyzed the electromechanical behavior of composite shell structures with embedded piezoelectric layers. To solve the equations, they applied the 3D-shell model based on the shell elements of discrete double directors. They utilized the third shear deformation theory as the displacement field. Akbari Alashti et al. [14] carried out the thermoelastic analysis of a functionally graded double curved shell. They assumed that the material varied in the thickness direction based on power law and, also, the piezoelectric layers were embedded in the upper and lower surfaces of the structure. They implemented the GDQ method to discretize the governing equations. They investigated the effects of temperature difference, grading index of the material, etc. Behjat et al. [15] conducted the static and dynamic analyses of a functionally graded piezoelectric plate. They hypothesized that the structure was under electrical and mechanical loads. They used the first-order shear deformation theory and the Hamilton principle to derive the governing equation. To solve the equations, they applied the FEM. The effects of the materials, boundary conditions, etc. were examined.

Although considerable researches have been devoted to vibrations of double curved structures, less attention has been paid to vibrations of smart double curved structures in a thermal condition. In the present paper, the considered structures are composed of three 
main layers. They consist of a core made of the functionally graded carbon nanotube (FG-CNT) and two piezoelectric layers on the upper and lower surfaces. The rule of mixture approximates the mechanical properties of the FGM. Moreover, the electrical field was approximated as a linear function. The main focus of this study is on four types of elliptical, spherical, cycloidal, and toro circular shells and panels. These structures are assumed to be moderate thick shells, and the first-order shear deformation theory is also implemented. The developed model is then solved by using both $1 \mathrm{D}$ and $2 \mathrm{D}$ GDQ methods [14]. It should be noted that the $2 \mathrm{D}$ method is more complicated than the $1 \mathrm{D}$ mode. Based on the obtained numerical solution using GDQ methods, a parametric sensitivity analysis is conducted to show the effect of different parameters including mechanical and electrical boundary conditions, volume distribution, and thermal environment on vibrations of the considered structures.

\section{Mathematical modeling}

The geometric schematics and coordinate systems are shown in Figures $1-5$. The $\alpha$ and $\beta$ values correspond

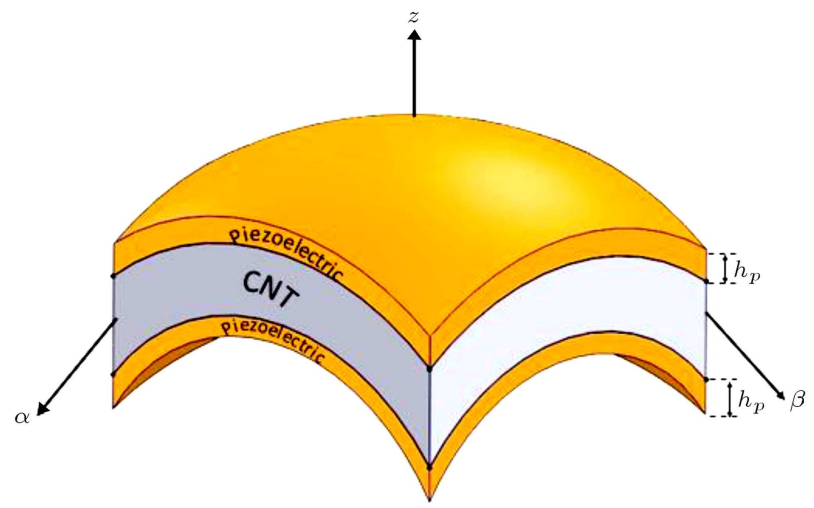

Figure 1. Coordinate system of doubly curved shell.

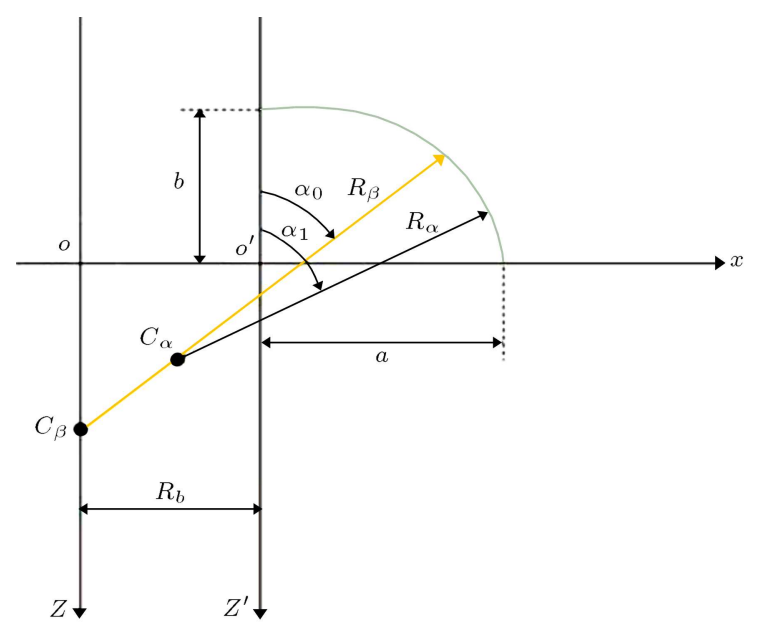

Figure 2. Coordinate system of elliptical shell.

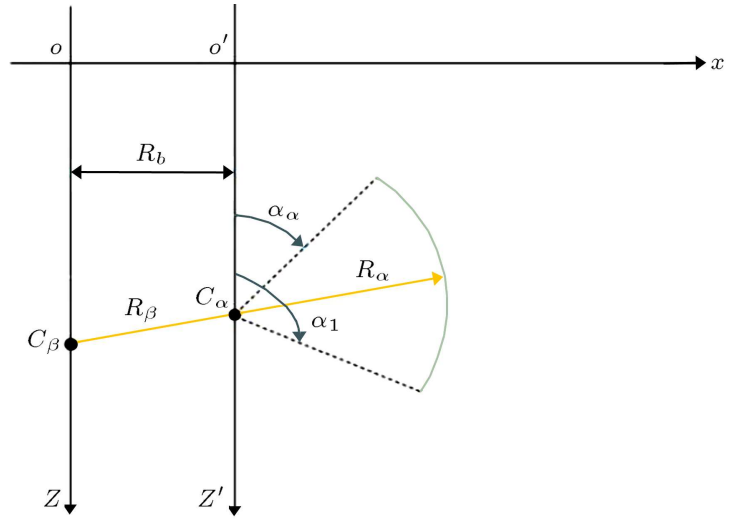

Figure 3. Coordinate system of cycloidal shell.

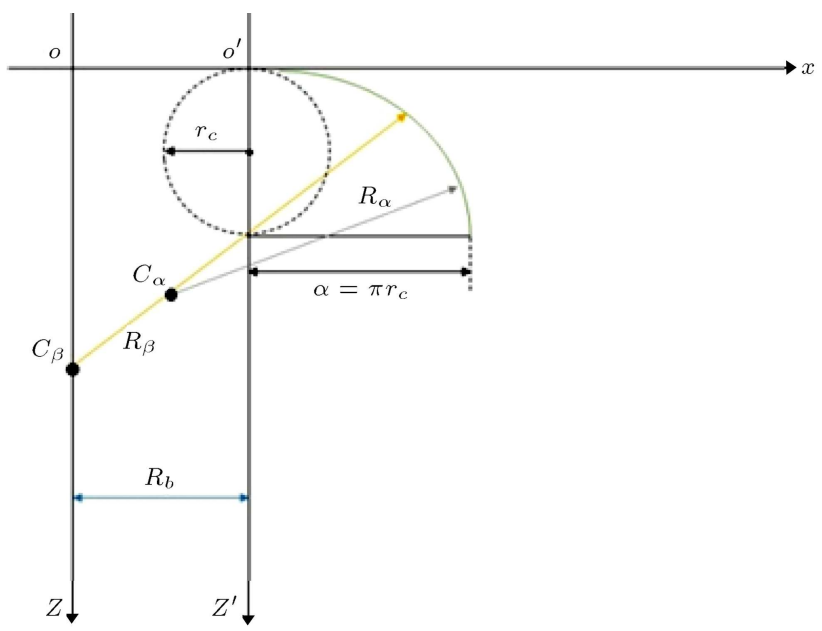

Figure 4. Coordinate system of toro circular shell.

to the meridional and circumferential coordinates. The position of any point in the shells is determined by $\alpha_{0}<$ $\alpha<\alpha_{1}, \beta_{0}<\beta<\beta_{1}$, and $-\frac{h}{2}<z<\frac{h}{2}$. The relation of the radii of structures is defined as follows [16]. In Figures 2-4, oz is known as the axis of revolution and $o^{\prime} z^{\prime}$ is the geometric axis of meridian. Further, the semi-major and semi-minor axes of the elliptical shell are denoted by $a$ and $b$, respectively. The distance between $o z$ and $o^{\prime} z^{\prime}$ is shown with $R_{b}$. The two radii of the structure are denoted by $R_{\alpha}$ and $R_{\beta}$.

Elliptical shell:

$$
\begin{aligned}
& R_{\alpha}=\frac{a^{2} b^{2}}{\sqrt{\left(a^{2} \sin ^{2}(\alpha)+b^{2} \cos ^{2}(\alpha)\right)^{3}}}, \\
& R_{\beta}=\frac{a^{2}}{\sqrt{\left(a^{2} \sin ^{2}(\alpha)+b^{2} \cos ^{2}(\alpha)\right)}}+\frac{R_{b}}{\sin (\alpha)} \\
& R_{0}=R_{2} \sin (\alpha) .
\end{aligned}
$$

Cycloidal shell:

$$
R_{0}=r_{c}(2 \alpha+\sin (2 \alpha)),
$$




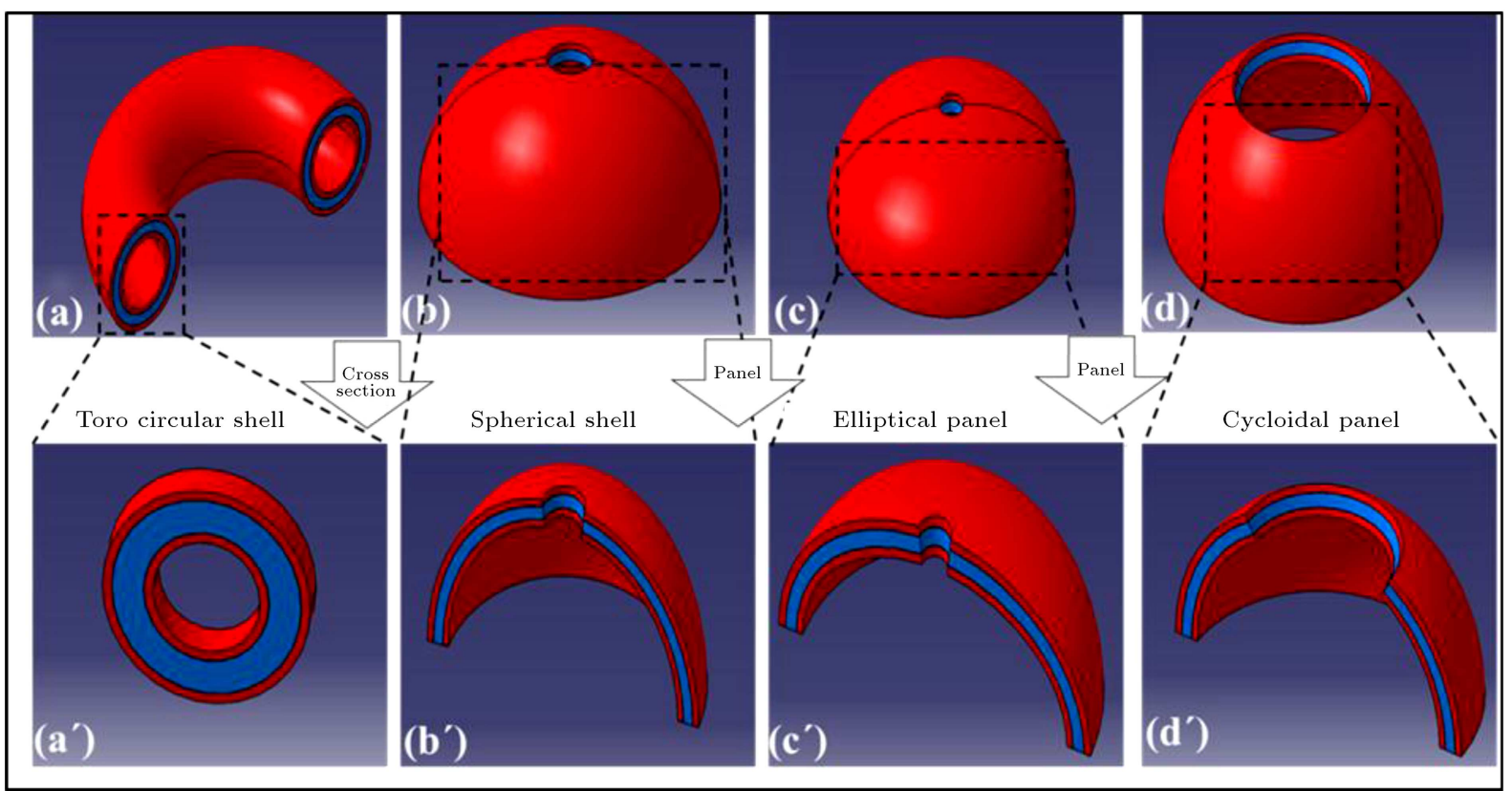

Figure 5. (a) Toro circular shell, (a') cross section of toro circular shell, (b) spherical shell, (b') spherical panel, (c) elliptical shell, ( $\left.c^{\prime}\right)$ elliptical panel, (d) cycloidal shell, and ( $\left.d^{\prime}\right)$ cycloidal panel.

$$
\begin{aligned}
R_{\alpha} & =4 r_{c} \cos (\alpha), \\
R_{\beta} & =\frac{R_{0}}{\sin (\alpha)} .
\end{aligned}
$$

Circular shell:

$$
\begin{aligned}
& R_{\alpha}=R, \\
& R_{0}=R \sin (\alpha)+R_{b}, \\
& R_{\beta}=\frac{R_{0}}{\sin (\alpha)} .
\end{aligned}
$$

In this research, based on the first-order shear deformation theory, the displacement fields are assumed below [17]:

$$
\begin{aligned}
& U(\alpha, \beta, z, t)=u(\alpha, \beta, t)+z \psi_{\alpha}(\alpha, \beta, t), \\
& V(\alpha, \beta, z, t)=v(\alpha, \beta, t)+z \psi_{\beta}(\alpha, \beta, t), \\
& W(\alpha, \beta, z, t)=w(\alpha, \beta, t) .
\end{aligned}
$$

In the above equations, $u, v$, and $w$ are displacements of the reference plane along the meridional, circumferential, and normal directions, respectively. $\psi_{\alpha}, \psi_{\beta}$ are the rotations around $\alpha$, and $\beta$ axes, respectively. It should be noted that in the above equations $U, V$ change linearly through the thickness and $W$ remains constant. Therefore, this assumption can be used for the small deflection theory. The general form of the strain-displacement relationships is represented as follows [17]:

$$
\begin{aligned}
\varepsilon_{i} & =\frac{\partial}{\partial \xi_{i}}\left(\frac{u_{i}}{A_{i}}\right)+\frac{1}{A_{i}} \sum_{k=1}^{3} \frac{u_{k}}{A_{k}} \frac{\partial A_{i}}{\partial \xi_{k}}, \\
\gamma_{i j} & =\frac{1}{A_{i} A_{j}}\left[A_{i}^{2} \frac{\partial}{\partial \xi_{j}}\left(\frac{u_{i}}{A_{i}}\right)+A_{j}^{2} \frac{\partial}{\partial \xi_{i}}\left(\frac{u_{j}}{A_{j}}\right)\right] \quad i \neq j .
\end{aligned}
$$

If the above equations are extended, the relationships are developed below [17]:

$$
\begin{aligned}
\varepsilon_{1} & =\frac{1}{A_{1}}\left(\frac{\partial U}{\partial \xi_{1}}+\frac{1}{a_{2}} \frac{\partial a_{1}}{\partial \xi_{2}} V+\frac{a_{1}}{R_{1}} w\right), \\
\varepsilon_{2} & =\frac{1}{A_{2}}\left(\frac{\partial V}{\partial \xi_{2}}+\frac{1}{a_{1}} \frac{\partial a_{2}}{\partial \xi_{1}} U+\frac{a_{2}}{R_{2}} w\right), \\
\gamma_{12} & =\frac{A_{2}}{A_{1}} \frac{\partial}{\partial \xi_{1}}\left(\frac{V}{A_{2}}\right)+\frac{A_{1}}{A_{2}} \frac{\partial}{\partial \xi_{2}}\left(\frac{U}{A_{1}}\right), \\
\varepsilon_{3} & =\left(\frac{\partial W}{\partial z}\right) \\
\gamma_{23} & =\frac{1}{A_{2}}\left(\frac{\partial W}{\partial \xi_{2}}\right)+A_{2} \frac{\partial}{\partial z}\left(\frac{V}{A_{2}}\right), \\
\gamma_{13} & =\frac{1}{A_{1}}\left(\frac{\partial W}{\partial \xi_{1}}\right)+A_{1} \frac{\partial}{\partial z}\left(\frac{U}{A_{1}}\right) .
\end{aligned}
$$

In the above equations, $U, V$, and $W$ are the displacements substituted by Eqs. (4a)-(4c) and $A_{1}, A_{2}$, and $A_{3}$, are the constants defined by the following equations [18]: 


$$
\begin{aligned}
& A_{1}=a_{1}\left(1+\frac{z}{R_{1}}\right), \\
& A_{2}=a_{2}\left(1+\frac{z}{R_{2}}\right), \\
& A_{3}=a_{3}=1 .
\end{aligned}
$$

The ratio of thickness to radius is zero because it is assumed that the shells are moderately thick. With this assumption, the following parameters are defined [18]:

Plate:

$$
\begin{aligned}
& A_{1}=1, \\
& A_{2}=1 .
\end{aligned}
$$

Cylindrical shell:

$$
\begin{aligned}
& A_{1}=1, \\
& A_{2}=R .
\end{aligned}
$$

Double curved shell:

$$
\begin{aligned}
& A_{1}=R_{\alpha}, \\
& A_{2}=R_{\beta} \sin (\alpha) .
\end{aligned}
$$

By substituting Eqs. (4a)-(4c) into Eqs. (6a)-(6f), the relationship between strains and mid-surface displacements is represented below [17]:

$$
\begin{aligned}
& \varepsilon_{\alpha}=\frac{1}{A_{1}} \frac{\partial u}{\partial \alpha}+\frac{v}{A_{1} A_{2}} \frac{\partial A_{1}}{\partial \beta}+\frac{w}{R_{\alpha}}, \\
& \varepsilon_{\beta}=\frac{1}{A_{2}} \frac{\partial v}{\partial \beta}+\frac{u}{A_{1} A_{2}} \frac{\partial A_{2}}{\partial \alpha}+\frac{w}{R_{\beta}}, \\
& \gamma_{\alpha \beta}=\frac{A_{2}}{A_{1}} \frac{\partial}{\partial \alpha}\left(\frac{v}{A_{2}}\right)+\frac{A_{1}}{A_{2}} \frac{\partial}{\partial \beta}\left(\frac{u}{A_{1}}\right),
\end{aligned}
$$

$$
\begin{aligned}
& \chi_{\alpha}=\frac{1}{A_{1}} \frac{\partial \psi_{\alpha}}{\partial \alpha}+\frac{\psi_{\beta}}{A_{1} A_{2}} \frac{\partial A_{1}}{\partial \beta}, \\
& \chi_{\beta}=\frac{1}{A_{2}} \frac{\partial \psi_{\beta}}{\partial \beta}+\frac{\psi_{\alpha}}{A_{1} A_{2}} \frac{\partial A_{2}}{\partial \alpha}, \\
& \chi_{\alpha \beta}=\frac{A_{2}}{A_{1}} \frac{\partial}{\partial \alpha}\left(\frac{\psi_{\beta}}{A_{2}}\right)+\frac{A_{1}}{A_{2}} \frac{\partial}{\partial \beta}\left(\frac{\psi_{\alpha}}{A_{1}}\right), \\
& \gamma_{\alpha z}=\psi_{\alpha}-\frac{u}{R_{\alpha}}+\frac{1}{A_{1}} \frac{\partial w}{\partial \alpha}, \\
& \gamma_{\beta z}=\psi_{\beta}-\frac{v}{R_{\beta}}+\frac{1}{A_{2}} \frac{\partial w}{\partial \beta} .
\end{aligned}
$$

In the above equations, $\varepsilon_{\alpha}, \varepsilon_{\beta}$, and $\gamma_{\alpha \beta}$ are strains in the meridional and circumferential directions and shearing elements, respectively, $\chi_{\alpha}, \chi_{\beta}$, and $\chi_{\alpha \beta}$ are the analogous curvature alterations. The material of the shells varies in the thickness direction based on the FGM model, and their strain relationships are linear. Therefore, the constitutive equations are shown below [19]. It should be noted that because of the piezoelectric properties and the presence of the thermal environment, resultant forces are developed by Eqs. (12a) and (12b) as shown in Box I.

In Eqs. (12a) and (12b), $\kappa$ is the factor of shear correction being assumed as 5/6. $N_{\alpha}, N_{\beta}$, and $N_{\alpha \beta}$ are resultant forces in the meridional, circumferential, and in-plain shearing parameters, respectively. $M_{\alpha}, M_{\beta}$, and $M_{\alpha \beta}$ are the resultant moments, and $Q_{\alpha}$ and $Q_{\beta}$ are the transverse shear force outcomes. The resultant piezoelectric and thermal effects are denoted by $p$ and $T$ indices, respectively. $D_{\alpha}, D_{\beta}$, and $D_{\alpha \beta}$ are the electrical displacements and $k_{i j}$ is the dielectric permittivity. $p_{i j}$ is the pyroelectric property, $e_{i j}$ is the piezoelectric stress constant, and $E_{i}$ is the component of the electrical field. The extensional stiffness $A_{i j}$,

$$
\begin{aligned}
& {\left[\begin{array}{c}
N_{\alpha} \\
N_{\beta} \\
N_{\alpha \beta} \\
M_{\alpha} \\
M_{\beta} \\
M_{\alpha \beta} \\
Q_{\alpha} \\
Q_{\beta}
\end{array}\right]=\left[\begin{array}{cccccccc}
A_{11} & A_{12} & 0 & B_{11} & B_{12} & 0 & 0 & 0 \\
A_{12} & A_{22} & 0 & B_{12} & B_{22} & 0 & 0 & 0 \\
0 & 0 & A_{66} & 0 & 0 & B_{66} & 0 & 0 \\
B_{11} & B_{12} & 0 & D_{11} & D_{12} & 0 & 0 & 0 \\
B_{12} & B_{22} & 0 & D_{12} & D_{22} & 0 & 0 & 0 \\
0 & 0 & B_{66} & 0 & 0 & D_{66} & 0 & 0 \\
0 & 0 & 0 & 0 & 0 & 0 & \kappa A_{55} & 0 \\
0 & 0 & 0 & 0 & 0 & 0 & 0 & \kappa A_{44}
\end{array}\right]\left[\begin{array}{c}
\varepsilon_{\alpha} \\
\varepsilon_{\beta} \\
\gamma_{\alpha \beta} \\
\chi_{\alpha} \\
\chi_{\beta} \\
\chi_{\alpha \beta} \\
\gamma_{\alpha z} \\
\gamma_{\beta z}
\end{array}\right]-\left[\begin{array}{c}
N_{\alpha}^{p} \\
N_{\beta}^{p} \\
0 \\
M_{\alpha}^{p} \\
M_{\beta}^{p} \\
0 \\
Q_{\alpha}^{p} \\
Q_{\beta}^{p}
\end{array}\right]\left[\begin{array}{c}
N_{\alpha}^{T} \\
N_{\beta}^{T} \\
0 \\
M_{\alpha}^{T} \\
M_{\beta}^{T} \\
0 \\
0 \\
0
\end{array}\right],} \\
& \left\{\begin{array}{l}
D_{\alpha} \\
D_{\beta} \\
D_{z}
\end{array}\right\}=\left[\begin{array}{ccccc}
0 & 0 & 0 & e_{15} & 0 \\
0 & 0 & e_{24} & 0 & 0 \\
e_{31} & e_{32} & 0 & 0 & 0
\end{array}\right]\left\{\begin{array}{c}
\varepsilon_{\alpha} \\
\varepsilon_{\beta} \\
\gamma_{\beta z} \\
\gamma_{\alpha z} \\
\gamma_{\alpha \beta}
\end{array}\right\}+\left[\begin{array}{ccc}
k_{11} & 0 & 0 \\
0 & k_{22} & 0 \\
0 & 0 & k_{33}
\end{array}\right]\left\{\begin{array}{l}
E_{\alpha} \\
E_{\beta} \\
E_{z}
\end{array}\right\}+\Delta T\left\{\begin{array}{l}
p_{\alpha} \\
p_{\beta} \\
p_{z}
\end{array}\right\}
\end{aligned}
$$


the bending stiffness $D_{i j}$, and the bending-extensional coupling stiffness $B_{i j}$ are defined below:

$$
\begin{aligned}
(A, B, D)= & \int_{-\frac{h}{2}}^{\frac{h}{2}} Q^{c}\left(1, z, z^{2}\right) d z+\int_{-\frac{h}{2}-h_{p}}^{-\frac{h}{2}} Q^{p} \\
& \left(1, z, z^{2}\right) d z+\int_{\frac{h}{2}}^{\frac{h}{2}+h_{p}} Q^{p}\left(1, z, z^{2}\right) d z .
\end{aligned}
$$

In the above relations, ' $c$ ' and ' $p$ ' represent core and piezoelectric parts, respectively.

$$
\begin{aligned}
& -\frac{h}{2} \leq z_{c} \leq \frac{h}{2}, \\
& -\frac{h}{2}-h_{p} \leq z_{p} \leq-\frac{h}{2}, \\
& \frac{h}{2} \leq z_{p} \leq \frac{h}{2}+h_{p} .
\end{aligned}
$$

It is worth noting that piezoelectric layers are substituted in the bottom and top layers. The components of the electrical field are defined below [20]:

$$
\begin{aligned}
& \left(E_{\alpha}^{t}, E_{\beta}^{t}, E_{z}^{t}\right)= \\
& \quad-\left(\frac{1}{A_{1}} \frac{2 z-h}{2 h_{p}} \frac{\partial \phi^{t}}{\partial \alpha}, \frac{1}{A_{2}} \frac{2 z-h}{2 h_{p}} \frac{\partial \phi^{t}}{\partial \beta}, \frac{1}{h_{p}} \phi^{t}\right), \\
& \left(E_{\alpha}^{b}, E_{\beta}^{b}, E_{z}^{b}\right)= \\
& \quad-\left(\frac{1}{A_{1}} \frac{2 z+h}{2 h_{p}} \frac{\partial \phi^{b}}{\partial \alpha}, \frac{1}{A_{2}} \frac{2 z+h}{2 h_{p}} \frac{\partial \phi^{b}}{\partial \beta}, \frac{1}{h_{p}} \phi^{b}\right) .
\end{aligned}
$$

In the above equation, $\phi$ is the electric potential that is equal to zero as follows:

$$
\alpha=\alpha_{1}, \alpha=\alpha_{2}, \quad \beta=\beta_{1}, \beta=\beta_{2} .
$$

Elastic constants of the core and layers are represented below [21]:

$$
\begin{aligned}
Q_{11} & =\frac{E_{11}}{1-\nu_{12} \nu_{21}}, \\
Q_{12} & =\frac{\nu_{21} E_{11}}{1-\nu_{12} \nu_{21}}, \\
Q_{22} & =\frac{E_{22}}{1-\nu_{12} \nu_{21}}, \\
Q_{66} & =G_{12}, \\
Q^{C} & =\left[\begin{array}{ccc}
Q_{11} & Q_{12} & 0 \\
Q_{21} & Q_{22} & 0 \\
0 & 0 & Q_{66}
\end{array}\right], \\
Q_{11}^{P} & =\frac{E_{P}}{1-\nu_{P}^{2}}, \\
Q_{12}^{P} & =\frac{\nu_{p} E_{P}}{1-\nu_{P}^{2}},
\end{aligned}
$$

$$
\begin{aligned}
Q_{11}^{p} & =Q_{22}^{p}, \\
Q_{66}^{p} & =\frac{E_{p}}{2\left(1+\nu_{p}\right)}, \\
Q^{p} & =\left[\begin{array}{ccc}
Q_{11}^{p} & Q_{12}^{p} & 0 \\
Q_{12}^{p} & Q_{22}^{p} & 0 \\
0 & 0 & Q_{66}^{p}
\end{array}\right] .
\end{aligned}
$$

Resultant force and momentum for the core and layers are represented as follows [21]:

$$
\begin{aligned}
\left(N^{T}, M^{T}, F^{T}\right)= & \int_{\frac{-h}{2}}^{\frac{h}{2}} Q^{c}\left(\begin{array}{c}
\alpha_{11} \\
\alpha_{22} \\
0
\end{array}\right)\left(1, z, z^{2}\right) \Delta T d z \\
& +\int_{\frac{-h}{2}-h_{p}}^{\frac{-h}{2}} Q^{p}\left(\begin{array}{c}
\alpha_{11}^{p} \\
\alpha_{22}^{p} \\
0
\end{array}\right) \Delta T d z \\
& +\int_{\frac{h}{2}}^{\frac{h}{2}+h_{p}} Q^{p}\left(\begin{array}{c}
\alpha_{11}^{p} \\
\alpha_{22}^{p} \\
0
\end{array}\right) \Delta T d z,
\end{aligned}
$$

$\left(N_{\alpha}^{p}, M_{\alpha}^{p}\right)=\int_{-\frac{h}{2}-h_{p}}^{-\frac{h}{2}}(1, z) e_{31} E_{z}^{b} d z+\int_{\frac{h}{2}}^{\frac{h}{2}+h_{p}}(1, z) e_{31} E_{z}^{t} d z$,

$$
\left(N_{\beta}^{p}, M_{\beta}^{p}\right)=\int_{-\frac{h}{2}-h_{p}}^{-\frac{h}{2}}(1, z) e_{31} E_{z}^{b} d z+\int_{\frac{h}{2}}^{\frac{h}{2}+h_{p}}(1, z) e_{31} E_{z}^{t} d z,
$$$$
\left(Q_{\alpha}^{p}\right)=\int_{-\frac{h}{2}-h_{p}}^{-\frac{h}{2}} e_{15} E_{\alpha}^{b} d z+\int_{\frac{h}{2}}^{\frac{h}{2}+h_{p}} e_{15} E_{\alpha}^{t} d z,
$$

$$
\left(Q_{\beta}^{p}\right)=\int_{-\frac{h}{2}-h_{p}}^{-\frac{h}{2}} e_{24} E_{\beta}^{b} d z+\int_{\frac{h}{2}}^{\frac{h}{2}+h_{p}} e_{24} E_{\beta}^{t} d z .
$$

As mentioned before, the core of the structures is made of FG-CNTs and its mechanical properties are defined below [22]:

$$
\begin{aligned}
& V_{c n t}(z)=V^{*}(U D), \\
& V_{c n t}(z)=2\left(1-\frac{2|z|}{h}\right) V^{*}(F G-O), \\
& V_{c n t}(z)=\frac{4|z|}{h} V^{*}(F G-x), \\
& V_{c n t}(z)=\left(1+\frac{2(z)}{h}\right) V^{*}(F G-V) .
\end{aligned}
$$




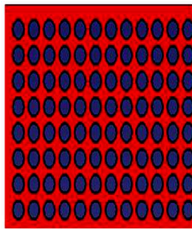

(a)

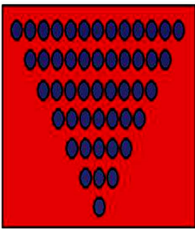

(b)

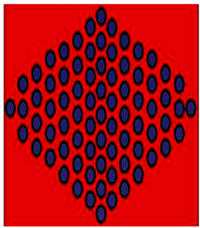

(c)

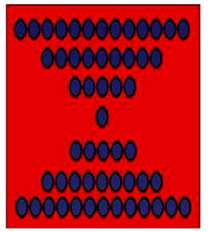

(d)
Figure 6. Schematic of Carbon Nanotube (CNT) dispersion in structures: (a) UD, (b) FGV, (c) FGO, and (d) FGX.

In the following, the schematic of the CNT dispersion is shown in a matrix [23]. In the above equations, $h$ is the thickness of structures and $z$ denotes the direction of thickness. The total volume fraction is denoted by $V^{*}$. In the UD (uniformly distributed) type model (Figure 6(a)), the distribution of CNT layer in the thickness direction remains stable. In the FGV model (Figure 6(b)), the higher and lower surfaces have the maximum and minimum distributions of CNT, respectively. The FGO model (Figure 6(c)) allocates maximum CNT to the center of the section and, thus, it becomes free of CNT on the top and bottom surfaces. The FGX model (Figure 6(d)) has the highest distribution of CNT on the top and bottom surfaces and without CNT on the middle plane. The mechanical properties of materials are defined below [22]:

$$
\begin{aligned}
E_{11} & =\eta_{1} V_{c n t}(z) E_{11}^{c n t}+V_{m}(z) E^{m} \\
\frac{\eta_{2}}{E_{22}}= & \frac{V_{c n t}(z)}{E_{22}^{c n t}}+\frac{V_{m}(z)}{E^{m}} \\
\nu_{12}= & V^{*} \nu_{12}^{c n t}+V_{m} \nu^{m} \\
\nu_{21}= & \nu_{12} \frac{E_{22}}{E_{11}}, \\
\frac{\eta_{3}}{G_{12}}= & \frac{V_{c n t}(z)}{G_{12}^{c n t}}+\frac{V_{m}(z)}{G^{m}}, \\
\rho(z)= & V_{c n t}(z) \rho^{c n t}+V_{m}(z) \rho^{m}, \\
\alpha_{11}= & \frac{V_{c n t}(z) E_{11}^{c n t} \alpha_{11}^{c n t}+V_{m}(z) E^{m} \alpha^{m}}{V_{c n t}(z) E_{11}^{c n t}+V_{m}(z) E^{m}}, \\
\alpha_{22}= & \left(1+\nu_{12}^{c n t}\right) V_{c n t}(z) \alpha_{22}^{c n t} \\
& +\left(1+\nu^{m}\right) V_{m}(z) \alpha^{m}-\nu_{12} \alpha_{11} .
\end{aligned}
$$

In the above equations, $\eta_{1}, \eta_{2}$, and $\eta_{3}$ are the first, second, and third parameters, respectively, which are all related to CNTs material. $E_{11}^{c n t}, E_{22}^{c n t}$, and $G_{12}^{c n t}$ are the young moduli and shear modulus of Single Wall Carbon Nanotubes (SWCNTs), respectively. $\rho^{\text {cnt }}$ and $\rho^{m}$ are mass densities of the CNT and matrix constituents, respectively. Moreover, $\alpha_{11}$ and $\alpha_{22}$ are the terms for thermal expansion coefficients in $\alpha$ and $\beta$ directions.

In order to derive the equation of motion of the considered structure, Hamilton's principle is implemented [24]:

$$
\int_{0}^{t}\left(\delta U_{\text {total }}+\delta V_{\text {total }}-\delta K_{\text {total }}\right) d t=0 .
$$

In the above formula, $\delta U, \delta V$, and $\delta K$ are the virtual strain energy, the virtual potential energy, and virtual kinetic energy, respectively.

$$
\begin{aligned}
\delta U_{\text {total }}= & \int_{A}\left(\sigma_{\alpha} \delta \varepsilon_{\alpha}+\sigma_{\beta} \delta \varepsilon_{\beta}+\sigma_{\alpha \beta} \delta \gamma_{\alpha \beta}+\kappa \sigma_{\alpha z} \delta \gamma_{\alpha z}\right. \\
& \left.+\kappa \sigma_{\beta z} \delta \gamma_{\beta z}-D_{\alpha} \delta E_{\alpha}-D_{\beta} \delta E_{\beta}-D_{z} \delta E_{z}\right) \\
& A_{1} A_{2} d \alpha d \beta \\
\delta V_{\text {total }}= & \int_{A}\left(N_{\alpha}^{T}\left(\frac{1}{A_{1}^{2}} \frac{\partial U}{\partial \alpha} \delta \frac{\partial U}{\partial \alpha}\right)\right. \\
& +N_{\alpha}^{T}\left(\frac{1}{A_{1}^{2}} \frac{\partial V}{\partial \alpha} \delta \frac{\partial V}{\partial \alpha}\right)+N_{\alpha}^{T}\left(\frac{1}{A_{1}^{2}} \frac{\partial W}{\partial \alpha} \delta \frac{\partial W}{\partial \alpha}\right) \\
& +N_{\beta}^{T}\left(\frac{1}{A_{2}^{2}} \frac{\partial U}{\partial \beta} \delta \frac{\partial U}{\partial \beta}\right)+N_{\beta}^{T}\left(\frac{1}{A_{2}^{2}} \frac{\partial V}{\partial \beta} \delta \frac{\partial V}{\partial \beta}\right) \\
& \left.+N_{\beta}^{T}\left(\frac{1}{A_{2}^{2}} \frac{\partial W}{\partial \beta} \delta \frac{\partial W}{\partial \beta}\right)\right) A_{1} A_{2} d \alpha d \beta, \quad(21) \\
& \left.+\left(\frac{\partial W}{\partial t} \delta \frac{\partial W}{\partial t}\right)\right) A_{1} A_{2} d \alpha d \beta \\
\delta K_{\text {total }}= & \int_{A} \rho\left(\left(\frac{\partial U}{\partial t} \delta \frac{\partial U}{\partial t}\right)+\left(\frac{\partial V}{\partial t} \delta \frac{\partial V}{\partial t}\right)\right. \\
& (22) \\
&
\end{aligned}
$$

By using Hamilton's principle, the motions of equations are obtained below:

$$
\begin{gathered}
\frac{1}{A_{1} A_{2}} \frac{\partial}{\partial \alpha}\left(A_{2} N_{\alpha}\right)-\frac{1}{A_{1} A_{2}} \frac{\partial A_{2}}{\partial \alpha} N_{\beta}+\frac{1}{A_{2}} \frac{\partial N_{\alpha \beta}}{\partial \beta}+\frac{Q_{\alpha}}{R_{\alpha}} \\
=\frac{1}{A_{1} A_{2}} N_{\alpha}^{T} \frac{\partial}{\partial \alpha}\left(\frac{A_{2}}{A_{1}} \frac{\partial u}{\partial \alpha}\right)+\frac{1}{A_{1} A_{2}} N_{\beta}^{T} \frac{\partial}{\partial \beta} \\
\left(\frac{A_{1}}{A_{2}} \frac{\partial u}{\partial \beta}\right)+\frac{1}{A_{1} A_{2}} M_{\alpha}^{T} \frac{\partial}{\partial \alpha}\left(\frac{A_{2}}{A_{1}} \frac{\partial \psi_{\alpha}}{\partial \alpha}\right) \\
+\frac{1}{A_{1} A_{2}} M_{\beta}^{T} \frac{\partial}{\partial \beta}\left(\frac{A_{1}}{A_{2}} \frac{\partial \psi_{\beta}}{\partial \beta}\right)+I_{0} \ddot{u}+I_{1} \ddot{\psi} \alpha,(23 \mathrm{a}) \\
\frac{1}{A_{2}} \frac{\partial N_{\beta}}{\partial \beta}+\frac{1}{A_{1} A_{2}} \frac{\partial A_{2}}{\partial \alpha} N_{\alpha \beta}+\frac{1}{A_{1} A_{2}} \frac{\partial}{\partial \alpha}\left(\left(A_{2} N_{\alpha \beta}\right)+\frac{Q_{\beta}}{R_{\beta}}\right. \\
=\frac{1}{A_{1} A_{2}} N_{\alpha}^{T} \frac{\partial}{\partial \alpha}\left(\frac{A_{2}}{A_{1}} \frac{\partial v}{\partial \alpha}\right)+\frac{1}{A_{1} A_{2}} N_{\beta}^{T} \frac{\partial}{\partial \beta}\left(\frac{A_{1}}{A_{2}} \frac{\partial v}{\partial \beta}\right)
\end{gathered}
$$




$$
\begin{aligned}
& +\frac{1}{A_{1} A_{2}} M_{\alpha}^{T} \frac{\partial}{\partial \alpha}\left(\frac{A_{2}}{A_{1}} \frac{\partial \psi_{\beta}}{\partial \alpha}\right) \\
& +\frac{1}{A_{1} A_{2}} M_{\beta}^{T} \frac{\partial}{\partial \beta}\left(\frac{A_{1}}{A_{2}} \frac{\partial \psi_{\beta}}{\partial \beta}\right)+I_{0} \ddot{v}+I_{1} \ddot{\psi}_{\beta}, \\
& -\frac{N_{\alpha}}{R_{\alpha}}-\frac{N_{\beta}}{R_{\beta}}+\frac{1}{A_{1} A_{2}} \frac{\partial}{\partial \alpha}\left(Q_{\alpha} A_{2}\right)+\frac{1}{A_{2}} \frac{\partial Q_{\beta}}{\partial \beta} \\
& =\frac{1}{A_{1} A_{2}} N_{\alpha}^{T} \frac{\partial}{\partial \alpha}\left(\frac{A_{2}}{A_{1}} \frac{\partial w}{\partial \alpha}\right)+\frac{1}{A_{1} A_{2}} \\
& N_{\beta}^{T} \frac{\partial}{\partial \beta}\left(\frac{A_{1}}{A_{2}} \frac{\partial w}{\partial \beta}\right)+I_{0} \ddot{w} \\
& \frac{1}{A_{1} A_{2}} \frac{\partial}{\partial \alpha}\left(A_{2} M_{\alpha}\right)-\frac{1}{A_{1} A_{2}} \frac{\partial A_{2}}{\partial \alpha} M_{\beta}+\frac{1}{A_{2}} \frac{\partial M_{\alpha \beta}}{\partial \beta} \\
& -Q_{\alpha}=\frac{1}{A_{1} A_{2}} M_{\alpha}^{T} \frac{\partial}{\partial \alpha}\left(\frac{A_{2}}{A_{1}} \frac{\partial u}{\partial \alpha}\right) \\
& +\frac{1}{A_{1} A_{2}} M_{\beta}^{T} \frac{\partial}{\partial \beta}\left(\frac{A_{1}}{A_{2}} \frac{\partial u}{\partial \beta}\right) \\
& +\frac{1}{A_{1} A_{2}} F_{\alpha}^{T} \frac{\partial}{\partial \alpha}\left(\frac{A_{2}}{A_{1}} \frac{\partial \psi_{\alpha}}{\partial \alpha}\right) \\
& +\frac{1}{A_{1} A_{2}} F_{\beta}^{T} \frac{\partial}{\partial \beta}\left(\frac{A_{1}}{A_{2}} \frac{\partial \psi_{\beta}}{\partial \beta}\right)+I_{1} \ddot{u}+I_{2} \ddot{\psi}_{\alpha}, \\
& \frac{1}{A_{2}} \frac{\partial M_{\beta}}{\partial \beta}+\frac{1}{A_{1} A_{2}} \frac{\partial A_{2}}{\partial \alpha} M_{\alpha \beta}+\frac{1}{A_{1} A_{2}} \frac{\partial}{\partial \alpha}\left(A_{2} M_{\alpha \beta}\right) \\
& -Q_{\beta}=\frac{1}{A_{1} A_{2}} M_{\alpha}^{T} \frac{\partial}{\partial \alpha}\left(\frac{A_{2}}{A_{1}} \frac{\partial v}{\partial \alpha}\right)+\frac{1}{A_{1} A_{2}} M_{\beta}^{T} \frac{\partial}{\partial \beta} \\
& \left(\frac{A_{1}}{A_{2}} \frac{\partial v}{\partial \beta}\right)+\frac{1}{A_{1} A_{2}} F_{\alpha}^{T} \frac{\partial}{\partial \alpha}\left(\frac{A_{2}}{A_{1}} \frac{\partial \psi_{\beta}}{\partial \alpha}\right) \\
& +\frac{1}{A_{1} A_{2}} F_{\beta}^{T} \frac{\partial}{\partial \beta}\left(\frac{A_{1}}{A_{2}} \frac{\partial \psi_{\beta}}{\partial \beta}\right)+I_{1} \ddot{v}+I_{2} \ddot{\psi}_{\beta}, \\
& \frac{1}{R_{\alpha} R_{\beta}} \frac{\partial}{\partial \alpha}\left(\frac{1}{R_{\alpha}} \int_{\frac{h}{2}}^{\frac{h}{2}+h_{p}} D_{\alpha}\left(\frac{2 z-h}{2 h_{p}}\right) d z\right) \\
& +\frac{1}{R_{\alpha} R_{\beta}} \frac{\partial}{\partial \beta}\left(\frac{1}{R_{\beta}} \int_{\frac{h}{2}}^{\frac{h}{2}+h_{p}} D_{\beta}\left(\frac{2 z-h}{2 h_{p}}\right) d z\right) \\
& \frac{1}{R_{\alpha} R_{\beta}}-\frac{1}{R_{\alpha} R_{\beta}} \int_{\frac{h}{2}}^{\frac{h}{2}+h_{p}} \frac{D_{z}}{h_{p}} d z=0
\end{aligned}
$$

$$
\begin{gathered}
\frac{1}{R_{\alpha} R_{\beta}} \frac{\partial}{\partial \alpha}\left(\frac{1}{R_{\alpha}} \int_{\frac{h}{2}}^{\frac{h}{2}+h_{p}} D_{\alpha}\left(\frac{2 z-h}{2 h_{p}}\right) d z\right)+\frac{1}{R_{\alpha} R_{\beta}} \frac{\partial}{\partial \beta} \\
\left(\frac{1}{R_{\beta}} \int_{\frac{h}{2}}^{\frac{h}{2}+h_{p}} D_{\beta}\left(\frac{2 z-h}{2 h_{p}}\right) d z\right) \frac{1}{R_{\alpha} R_{\beta}} \\
-\frac{1}{R_{\alpha} R_{\beta}} \int_{\frac{h}{2}}^{\frac{h}{2}+h_{p}} \frac{D_{z}}{h_{p}} d z=0 .
\end{gathered}
$$

\section{Solution procedure}

\subsection{GDQ method in one dimension}

In this work, the GDQ method is applied to solve the equations of motion of the considered structures. In order to solve the equations, they are assumed constant in the circumferential direction. As a result, Eqs. (24a) $-(24 \mathrm{~g})$ are used to reduce $2 \mathrm{D}$ equations into $1 \mathrm{D}[25]$.

$$
\begin{aligned}
& u(\alpha, \beta, t)=u(\alpha, t) \cos (n \beta), \\
& v(\alpha, \beta, t)=v(\alpha, t) \sin (n \beta), \\
& w(\alpha, \beta, t)=w(\alpha, t) \cos (n \beta), \\
& \psi_{\alpha}(\alpha, \beta, t)=\psi_{\alpha}(\alpha, t) \cos (n \beta), \\
& \psi_{\beta}(\alpha, \beta, t)=\psi_{\beta}(\alpha, t) \sin (n \beta), \\
& \phi_{\alpha}(\alpha, \beta, t)=\phi_{\alpha}(\alpha, t) \cos (n \beta), \\
& \phi_{\beta}(\alpha, \beta, t)=\phi_{\beta}(\alpha, t) \sin (n \beta) .
\end{aligned}
$$

Based on this method, grid points along the reference surface are given as follows [26]:

$$
\alpha_{i}=\left(1-\cos \left(\frac{i-1}{N-1} \pi\right)\right) \frac{\left(\alpha_{1}-\alpha_{0}\right)}{2}+\alpha_{0} .
$$

The final equation of motion is approximated by using the 1D GDQ method. Details of this method are given below [26].

$$
\begin{aligned}
& \frac{\partial^{n} f}{\partial \alpha^{n}}=\sum_{k=1}^{N} c_{i k}^{n} f_{k}, \\
& M^{(1)}\left(\alpha_{k}\right)=\prod_{j=1, j \neq k}^{N}\left(\alpha_{k}-\alpha_{j}\right), \\
& c_{i j}^{1}=\frac{M^{(1)}\left(\alpha_{i}\right)}{\left(\alpha-\alpha_{j}\right) M^{(1)}\left(\alpha_{j}\right)} \\
& \quad i, j=1,2, \ldots, N, \quad j \neq i,
\end{aligned}
$$




$$
\begin{gathered}
c_{i j}^{n}=n\left(c_{i j}^{1} *\left(c_{i i}^{(n-1)}-c_{i j}^{(n-1)}\right) /\left(\alpha_{i}-\alpha_{j}\right)\right) \\
i, j=1,2, \ldots, j \neq i, \quad n=2,3, \ldots, N-1, \\
c_{i i}^{n}=-\sum_{j=1, j \neq i}^{N} c_{i j}^{n} \\
i, j=1,2, \ldots, N, \quad n=1,2,3, \ldots, N-1 .
\end{gathered}
$$

For analyzing the shell structures, three boundary conditions are presumed being displayed in Table 1.

\subsection{GDQ method in two dimensions}

For 2D solution, the approximations are considered in the two following directions [4]:

$$
\begin{gathered}
\frac{\partial^{n} u}{\partial \alpha^{n}}=\sum_{k=1}^{N} c_{i k}^{n} u_{k j}, \\
\frac{\partial^{n} u}{\partial \beta^{n}}=\sum_{m=1}^{N} c_{j m}^{n} u_{i m} .
\end{gathered}
$$

Based on this method, grid points along the reference surface are considered as follows:

$$
\begin{aligned}
& \alpha_{i}=\left(1-\cos \left(\frac{i-1}{N-1} \pi\right)\right) \frac{\left(\alpha_{1}-\alpha_{0}\right)}{2}+\alpha_{0}, \\
& \beta_{j}=\left(1-\cos \left(\frac{j-1}{M-1} \pi\right)\right) \frac{\left(\beta_{1}-\beta_{0}\right)}{2}+\beta_{0} . \\
& M^{(1)}\left(\alpha_{k}\right)=\prod_{j=1, j \neq k}^{N}\left(\alpha_{k}-\alpha_{j}\right), \\
& M^{(1)}\left(\beta_{k}\right)=\prod_{j=1, j \neq k}^{N}\left(\beta_{k}-\beta_{j}\right),
\end{aligned}
$$

Table 1. Boundary conditions of the shell structures.

$$
\begin{array}{lcl}
\hline \text { C-C } & i=1, i=N & u_{i}=v_{i}=w_{i}=M_{\alpha i}=\psi_{\beta i}=0 \\
& & u_{i}=v_{i}=w_{i}=\psi_{\alpha i}=\psi_{\beta i}=0 \\
& & \\
\text { C-S } & i=1, i=N & u_{i}=v_{i}=w_{i}=\psi_{\alpha i}=\psi_{\beta i}=0 \\
& & u_{i}=v_{i}=w_{i}=M_{\alpha i}=\psi_{\beta i}=0 \\
& & \\
\text { S-S } & i=1, i=N & u_{i}=v_{i}=w_{i}=M_{\alpha i}=\psi_{\beta i}=0 \\
\hline
\end{array}
$$

$$
\begin{aligned}
c_{i j}^{1}= & \frac{M^{(1)}\left(\alpha_{i}\right)}{\left(\alpha-\alpha_{j}\right) M^{(1)}\left(\alpha_{j}\right)} \\
& i, j=1,2, \ldots, N, \quad j \neq i, \\
c p_{i j}^{1}= & \frac{M^{(1)}\left(\beta_{i}\right)}{\left(\beta-\beta_{j}\right) M^{(1)}\left(\beta_{j}\right)} \\
& i, j=1,2, \ldots, N, \quad j \neq i, \\
c_{i j}^{n}= & n\left(c_{i j}^{1} *\left(c_{i i}^{(n-1)}-c_{i j}^{(n-1)}\right) /\left(\alpha_{i}-\alpha_{j}\right)\right) \\
& i, j=1,2, \ldots, j \neq i, \quad n=2,3, \ldots, N-1, \\
c p_{i j}^{n}= & n\left(c p_{i j}^{1} *\left(c p_{i i}^{(n-1)}-c p_{i j}^{(n-1)}\right) /\left(\beta_{i}-\beta_{j}\right)\right) \\
& i, j=1,2, \ldots, j \neq i, \quad n=2,3, \ldots, N-1, \\
c p_{i i}^{n}= & \quad \sum_{j=1, j \neq i}^{N} c p_{i j}^{n}, j=1,2, \ldots, N, \quad n=1,2,3, \ldots, N-1 . \\
c_{i i}^{n}= & \sum_{j=1, j \neq i}^{N} c_{i j}^{n} \quad n=1,2,3, \ldots, N-1, \\
i, j=1,2, \ldots, N, \quad 2,2 &
\end{aligned}
$$

For the panel structures, the boundary conditions are considered as Table 2.

In the panel, for example, $\mathrm{C}-\mathrm{S}-\mathrm{C}-\mathrm{S}$ indicates that the panel is clamped at $\alpha=\alpha_{0}$ and $\alpha=\alpha_{1}$; thus, it is simply supported at $\beta=\beta_{0}$ and $\beta=\beta_{1}$.

By using the GDQ method, the matrix form of the equations of motion is as follows:

$$
\begin{aligned}
& {[M]\{\ddot{m}\}+\left[K_{m m}\right]\{m\}+\left[K_{m E}\right]\{E\}=0,} \\
& {\left[K_{E m}\right]\{m\}+\left[K_{E E}\right]\{E\}=0 .}
\end{aligned}
$$

In the above equation, the following can be proposed:

$$
\begin{aligned}
& \{m\}=\left\{\begin{array}{lllll}
u & v & w & \psi_{\alpha} & \psi_{\beta}
\end{array}\right\}^{T}, \\
& \{E\}=\left\{\begin{array}{lll}
\phi_{b} & \phi_{t}
\end{array}\right\}^{T} .
\end{aligned}
$$

Indices of $b$ and $t$ indicate the bottom and top layers, respectively. By omitting the electrical variables,

Table 2. Bounday conditions of the panel structures.

\begin{tabular}{lll}
\hline C-C-C-C & $i=1,2, \ldots, M, j=1,2, \ldots, N$ & $u_{i j}=v_{i j}=w_{i j}=\psi_{\alpha i j}=\psi_{\beta i j}=0$ \\
& $i=1, M, j=1,2, \ldots N$ & $u_{i j}=v_{i j}=w_{i j}=M_{\alpha i j}=\psi_{\beta i j}=0$ \\
C-S-C-S & $i=1,2, \ldots M, j=1, N$ & $u_{i j}=v_{i j}=w_{i j}=\psi_{\alpha i j}=M_{\beta i j}=0$ \\
\hline
\end{tabular}


the final form of the equation will be introduced as follows [27]:

$$
[M]\{\ddot{m}\}+\left(\left[K_{m m}\right]-\left[K_{m E}\right]\left[K_{E E}\right]^{-1}\left[K_{E m}\right]\right)\{m\}=0 .
$$

To explore the effect of electrical properties, two boundary conditions including open and closed circuits will be considered. The electrical condition is closed if the upper and lower surfaces are ground; otherwise, it is open.

$$
[M]\{\ddot{m}\}+\left(\left[K_{m m}\right]\right)\{m\}=0 .
$$

Eq. (32) or (33) is separated into two equations:

$$
\begin{aligned}
& {\left[K_{d b}\right]\left\{m_{b}\right\}+\left[K_{d d}\right]\left\{m_{d}\right\}=[M]\left\{\ddot{m}_{d}\right\},} \\
& {\left[K_{b b}\right]\left\{m_{b}\right\}+\left[K_{b d}\right]\left\{m_{d}\right\}=0 .}
\end{aligned}
$$

After omitting $\left\{m_{b}\right\}$, the final form is as follows:

$$
\left\{\left[K_{d d}\right]-\left[K_{d b}\right]\left[K_{b b}\right]^{-1}\left[K_{b d}\right]\right\}\left\{m_{d}\right\}=M\left\{\ddot{m}_{d}\right\} .
$$

In the above equation, $b$ and $d$ indices present the boundary and inside domains, respectively. In Eqs. (32) and (33), $m_{b}$ and $m_{d}$ are defined as below:

For 1D solution:

$$
\begin{aligned}
& \left\{m_{b}\right\}=\left\{u_{1}, u_{N}, v_{1}, v_{N}, w_{1}, w_{N},\right. \\
& \left.\psi_{\alpha_{1}}, \psi_{\alpha_{N}}, \psi_{\beta_{1}}, \psi_{\beta_{N}}\right\} \\
& \left\{m_{d}\right\}=\left\{u_{2}, u_{3}, \ldots ., u_{N-1}, v_{2}, v_{3}, \ldots ., v_{N-1},\right. \\
& w_{2}, w_{3}, \ldots ., w_{N-1}, \psi_{\alpha_{2}}, \psi_{\alpha_{3}}, \ldots ., \psi_{\alpha_{N-1}}, \psi_{\beta_{2}}, \\
& \left.\psi_{\beta_{3}}, \ldots ., \psi_{\beta_{N-1}}\right\} .
\end{aligned}
$$

For 2D solution:

$$
\begin{aligned}
\left\{m_{d a}\right\}= & \left\{a_{22}, a_{23}, \ldots, a_{2 N-1}, a_{32}, a_{33}, \ldots, a_{3 N-1}, \ldots,\right. \\
& \left.a_{(N-1) 1}, a_{(N-1) 2}, \ldots, a_{(N-1)(N-1)}\right\}^{T},
\end{aligned}
$$

$$
\begin{aligned}
& a=\left\{u, v, w, \psi_{\alpha}, \psi_{\beta}\right\} \\
&\left\{m_{d}\right\}=\left\{m_{d u}, m_{d v}, m_{d w}, m_{d \psi_{\alpha}}, m_{d \psi_{\beta}}\right\}^{T}, \\
&\left\{m_{b a}\right\}=\left\{a_{11}, a_{12}, \ldots, a_{1 N}, a_{21}, a_{2 N}, \ldots,\right. \\
&\left.a_{(N-1) 1}, a_{(N-1) N}, a_{N 1}, a_{N 2}, \ldots, a_{N N}\right\}, \\
&\left\{m_{b}\right\}=\left\{m_{b u}, m_{b v}, m_{b w}, m_{b \psi_{\alpha}}, m_{b \psi_{\beta}}\right\}^{T} .
\end{aligned}
$$

For free vibration, $\left\{m_{d}\right\}=m_{d} e^{i \omega t}$ is assumed. Therefore, the final form which provides vibration frequency is given below:

$$
\left(\left\{\left[K_{d d}\right]-\left[K_{d b}\right]\left[K_{b b}\right]^{-1}\left[K_{b d}\right]\right\}+[M] \omega^{2}\right)\left\{m_{d}\right\}=0 .
$$

\section{Numerical analysis and discussion}

In the current study, the free vibration responses of the FG-CNT double-curved shells and panels embedded in piezoelectric layers are studied. At the first stage, results are compared to those of other studies. Polymethyl methacrylate is chosen as a matrix with the following material properties [28].

$$
\begin{aligned}
& E_{m}=\left(3.52-0.003^{*} \Delta T\right) \mathrm{GPa}, \\
& \nu_{m}=0.34 \quad \rho=1150 \mathrm{~kg} / \mathrm{m}^{3} .
\end{aligned}
$$

Here, $\Delta T=T_{0}$. The mechanical properties of SWCNT at different temperatures are given in Tables 3 and 4. Also, the material characteristics of PZT-5A are displayed in Table 5.

For validation, data results are initially obtained from shells and panels via the GDQ method and, then, they are compared with those of other works. To investigate validity, the materials including zirconia (ceramic) and aluminum (metal) are added to the FGM model. Young's modulus, Poisson's ratio, and mass density are: $E_{c}=168 \mathrm{GPa}, \nu=0.3$, and $\rho_{c}=5700 \mathrm{~kg} / \mathrm{m}^{3}$, respectively, for zirconia; they are

Table 3. Thermo mechanical properties of (10.10) armchair Single Wall Carbonnano Tube (SWCNT) at some specific temperatures [31].

\begin{tabular}{cccccccc}
\hline $\boldsymbol{T}$ & $\boldsymbol{E}_{\mathbf{1 1}}^{C N T}$ & $\boldsymbol{\alpha}_{\mathbf{2 2}}^{C N T} * \mathbf{1 0}^{-\mathbf{6}}\left(\frac{\mathbf{1}}{\mathrm{c}}\right)$ & $\begin{array}{c}\boldsymbol{E}_{\mathbf{2 2}}^{C N T} \\
(\mathbf{T P a})\end{array}$ & $\boldsymbol{\alpha}_{\mathbf{1 1}}^{C N T} * \mathbf{1 0}^{-\mathbf{6}}\left(\frac{\mathbf{1}}{\mathrm{c}}\right)$ & $\begin{array}{c}\boldsymbol{G}_{\mathbf{1 2}}^{C N T} \\
(\mathbf{T P a})\end{array}$ & $\boldsymbol{\nu}_{\mathbf{1 2}}^{C N T}$ & $\begin{array}{c}\boldsymbol{\rho} \\
\left(\frac{\mathrm{kg}}{\mathbf{m}^{3}}\right)\end{array}$ \\
\hline 300 & 5.64 & 5.16 & 7.08 & 3.45 & 1.94 & 0.17 & 1400 \\
500 & 5.53 & 5.01 & 6.93 & 4.53 & 1.96 & 0.17 & 1400 \\
700 & 5.47 & 5.01 & 6.86 & 4.53 & 1.96 & 0.17 & 1400 \\
\hline
\end{tabular}


Table 4. The parameters of $(10,10)$ armchair Single Wall Carbonnano Tube (SWCNT) obtained from molecular dynamics [32].

\begin{tabular}{cccc}
\hline $\boldsymbol{V}_{\boldsymbol{C N \boldsymbol { T }}}^{*}$ & $\boldsymbol{\eta}_{\mathbf{1}}$ & $\boldsymbol{\eta}_{\mathbf{2}}$ & $\boldsymbol{\eta}_{\mathbf{3}}$ \\
\hline 0.12 & 0.137 & 1.022 & $0.7 \eta_{2}$ \\
0.17 & 0.142 & 1.626 & $0.7 \eta_{2}$ \\
0.18 & 0.141 & 1.585 & $0.7 \eta_{2}$ \\
\hline
\end{tabular}

$E_{m}=70 \mathrm{GPa}, v_{m}=0.3$, and $\rho_{m}=2707 \mathrm{~kg} / \mathrm{m}^{3}$, respectively, for aluminum. The volume fraction of FGM is considered as:

$F G M_{I}(a / b / c / p): V_{1}=\left[1-a\left(\frac{1}{2}+\frac{z}{h}\right)+b\left(\frac{1}{2}+\frac{z}{h}\right)^{c}\right]^{p}$,

$F G M_{I I}(a / b / c / p): V_{2}=\left[1-a\left(\frac{1}{2}-\frac{z}{h}\right)+b\left(\frac{1}{2}-\frac{z}{h}\right)^{c}\right]^{p}$.

It is worth noting that for validation, the spherical shell is considered and the results are compared to those found in [29]. The geometrical properties of the spherical shell are assumed as follows:

$$
\varphi_{0}=\frac{\pi}{8}, \quad \varphi_{1}=\frac{\pi}{2}, \quad h=0.05, \quad R=1 .
$$

The results for this purpose are provided in Table 6 .

It should be noted that these results are obtained without piezoelectric materials and at room temperature. For validation of the panel analysis, the toro circular panel is analyzed. Then, the results were compared with those found in [30]. The obtained results are explained in Table 7 . For this purpose, the materials are assumed as FGM and the volume fraction is given below:

$$
F G M_{I}=\left(\frac{1}{2}-\frac{z}{h}\right)^{p}, \quad F G M_{I I}=\left(\frac{1}{2}+\frac{z}{h}\right)^{p} .
$$

Moreover, the geometrical properties of this structure are given below:

$$
\begin{aligned}
& R=3, \quad h=0.2, \quad \varphi_{0}=\frac{\pi}{3}, \quad \varphi_{1}=\frac{2 \pi}{3}, \quad \vartheta=\frac{2 \pi}{3}, \\
& R_{b}=1.5, \quad D_{m}=\frac{E_{m} h^{3}}{12\left(1-\nu_{m}^{2}\right)} .
\end{aligned}
$$

Table 5. Mechanical and electrical properties of PZT-5A [33].

\begin{tabular}{|c|c|c|c|c|c|}
\hline \multirow{2}{*}{$\boldsymbol{P}$} & \multirow{2}{*}{$n$} & \multicolumn{2}{|c|}{ S-S } & \multicolumn{2}{|c|}{ C-C } \\
\hline & & Present & Ref. [29] & Present & Ref. [29 \\
\hline \multirow{3}{*}{$F G M_{I}(a=1 / b=0 / c / p=0.6)$} & 1 & 827.82 & 827.89 & 910.55 & 910.69 \\
\hline & 2 & 886.88 & 886.9 & 849.65 & 894.68 \\
\hline & 3 & 881.63 & 881.64 & 889.43 & 889.45 \\
\hline \multirow{3}{*}{$p=20$} & 1 & 797.35 & 797.41 & 887 & 887.13 \\
\hline & 2 & 851.91 & 851.92 & 859 & 859.37 \\
\hline & 3 & 848.52 & 848.53 & 856.54 & 856.57 \\
\hline \multirow{3}{*}{$F G M_{I I}(a=1 / b=0 / c=1 / p=0.6)$} & 1 & 815.15 & 815.21 & 908.66 & 908.8 \\
\hline & 2 & 887.92 & 887.95 & 893.78 & 893.81 \\
\hline & 3 & 885.54 & 885.56 & 888.30 & 888.32 \\
\hline \multirow{3}{*}{$p=20$} & 1 & 790.75 & 790.81 & 885.80 & 885.93 \\
\hline & 2 & 852.66 & 852.68 & 858.73 & 858.76 \\
\hline & 3 & 850.57 & 850.59 & 855.74 & 855.76 \\
\hline
\end{tabular}

\begin{tabular}{ccccc}
\hline E (GPa) & $\boldsymbol{v}$ & $\boldsymbol{\rho}\left(\frac{\mathrm{kg}}{\mathrm{m}^{3}}\right)$ & $\begin{array}{c}\text { Electro mechanical } \\
\left(\frac{\mathrm{c}}{\mathrm{m}^{3}}\right)\end{array}$ & $\begin{array}{c}\text { Permittivity coefficient } \\
\left(\frac{\boldsymbol{F}}{\mathbf{m}} \times \mathbf{1 0}^{-\mathbf{8}}\right)\end{array}$ \\
\hline \multirow{3}{*}{63} & \multirow{3}{*}{0.35} & \multirow{2}{*}{7600} & $e_{31}=-7.29$ & $K_{11}=1.53$ \\
& & $e_{32}=-7.29$ & $K_{22}=1.53$ \\
& & $e_{15}=12.322$ & $K_{33}=1.5$ \\
& & $e_{24}=12.322$ & \\
\hline
\end{tabular}

Table 6. Comparison of frequencies (HZ) for the Functionally Graded Material (FGM) spherical shell with different boundary conditions. Geometrical properties of the spherical shell are: $\varphi_{0}=\frac{\pi}{8}, \varphi_{1}=\frac{\pi}{2}, h=0.05, R=1$. 
Table 7. Comparison of frequency parameters $\Omega=\omega R^{2} \sqrt{\frac{\rho_{m} h}{D_{m}}}$ for Functionally Graded Material (FGM) toro circular panel. Geometrical properties of this structure are: $R=3, h=0.2, \varphi_{0}=\frac{\pi}{3}, \varphi_{1}=\frac{2 \pi}{3}, \vartheta=\frac{2 \pi}{3}, R_{b}=1.5$.

\begin{tabular}{|c|c|c|c|c|c|c|}
\hline \multirow{2}{*}{ Type } & \multirow{2}{*}{$\vartheta$} & \multirow{2}{*}{$P$} & \multicolumn{2}{|c|}{$\mathrm{CCCC}$} & \multicolumn{2}{|c|}{ CSCS } \\
\hline & & & Present & Ref. [29] & Present & Ref. [30] \\
\hline \multirow{6}{*}{$F G M_{I}$} & \multirow{2}{*}{$\frac{\varpi}{3}$} & 0.5 & 63.05 & 63.06 & 59.47 & 60.38 \\
\hline & & 10 & 60.48 & 61.41 & 58.08 & 59.06 \\
\hline & \multirow{2}{*}{$\frac{2 \pi}{3}$} & 0.5 & 56.90 & 57.77 & 56.66 & 57.53 \\
\hline & & 10 & 55.45 & 56.30 & 55.16 & 56.01 \\
\hline & \multirow{2}{*}{$\frac{4 \pi}{3}$} & 0.5 & 56.07 & 56.79 & 56.06 & 56.74 \\
\hline & & 10 & 54.53 & 55.26 & 54.52 & 55.23 \\
\hline \multirow{6}{*}{$F G M_{I I}$} & \multirow{2}{*}{$\frac{\pi}{3}$} & 0.5 & 62.00 & 62.95 & 59.79 & 60.71 \\
\hline & & 10 & 60.37 & 61.30 & 58.43 & 59.33 \\
\hline & \multirow{2}{*}{$\frac{2 \pi}{3}$} & 0.5 & 56.82 & 57.67 & 56.65 & 57.51 \\
\hline & & 10 & 55.34 & 56.18 & 55.13 & 55.96 \\
\hline & \multirow{2}{*}{$\frac{4 \pi}{3}$} & 0.5 & 55.99 & 56.69 & 55.99 & 56.65 \\
\hline & & 10 & 54.44 & 55.15 & 54.43 & 55.12 \\
\hline
\end{tabular}

To check the accuracy for the structures with piezoelectric materials, the results are obtained for a square plate. The plate is made of FG-CNT and embedded in the piezoelectric layer on the top and bottom surfaces. The results are reported in Table 8 . The piezoelectric material considered in this study is PZT-5A. Of note, the parameters of the plate structure are:

$$
A_{1}=1, \quad A_{2}=1, \quad R_{\alpha}=\infty, \quad R_{\beta}=\infty .
$$

Subsequently, by substituting these parameters into the governing equations, the results are obtained for the plate. The geometrical properties of the plate are given below:

$$
x_{1}=0.4, \quad y_{1}=0.4, \quad h=0.05, \quad h p=0.1 h
$$

\subsection{Case study result}

In this section, the effects of electrical and mechanical boundary conditions, the distribution of CNTs, thermal environment, the volume fraction of CNTs, and the geometrical characteristics of the shells and panels are studied. Tables 9-12 present the fundamental frequency of the spherical, toro circular, cycloidal, and elliptical shells that are reinforced by CNTs and integrated with piezoelectric layers. The natural frequencies of the four structures with three boundary conditions including $\mathrm{C}-\mathrm{C}, \mathrm{C}-\mathrm{S}$, and $\mathrm{S}-\mathrm{S}$ are shown in Tables 9-12. Based on these results, the switching of the electrical boundary conditions (shifting from closed circuit to open circuit) leads to increase in the amount of the fundamental frequencies. Following the comparison of the results of the CNTs pattern distributions, it becomes clear that the FG-X type of CNTs distribution has the highest frequency, while the FG-O pattern has the lowest frequency value. It
Table 8. Comparison of frequency parameters $\Omega=\omega \frac{x_{1}^{2}}{h} \sqrt{\frac{\rho_{m}}{E_{m}}}$ for CCCC plate integrated with PZT-5A layers. The geometrical properties of the plate are:

\begin{tabular}{|c|c|c|c|c|}
\hline$V_{C N T}^{*}$ & Type & Condition & \multicolumn{2}{|c|}{$\Omega$} \\
\hline \multirow{2}{*}{0.12} & \multirow{2}{*}{ FGO } & Open & 5.32 & 5.46 \\
\hline & & Close & 5.19 & 5.23 \\
\hline \multirow{2}{*}{0.17} & \multirow{2}{*}{ FGO } & Open & 5.60 & 5.72 \\
\hline & & Close & 5.47 & 5.52 \\
\hline \multirow{2}{*}{0.28} & \multirow{2}{*}{$\mathrm{FGO}$} & Open & 6.01 & 6.12 \\
\hline & & Close & 5.84 & 5.93 \\
\hline \multirow{2}{*}{0.12} & \multirow{2}{*}{ FGV } & Open & 5.54 & 5.67 \\
\hline & & Close & 5.40 & 5.45 \\
\hline \multirow{2}{*}{0.17} & \multirow{2}{*}{ FGV } & Open & 5.88 & 5.98 \\
\hline & & Close & 5.73 & 5.80 \\
\hline \multirow{2}{*}{0.28} & \multirow{2}{*}{ FGV } & Open & 6.34 & 6.63 \\
\hline & & Close & 6.16 & 6.28 \\
\hline \multirow{2}{*}{0.12} & \multirow{2}{*}{ FGX } & Open & 6.17 & 6.27 \\
\hline & & Close & 5.97 & 6.09 \\
\hline \multirow{2}{*}{0.17} & \multirow{2}{*}{ FGX } & Open & 6.68 & 6.78 \\
\hline & & Close & 5.46 & 6.62 \\
\hline \multirow{2}{*}{0.28} & \multirow{2}{*}{ FGX } & Open & 7.33 & 7.42 \\
\hline & & Close & 7.06 & 7.29 \\
\hline \multirow{2}{*}{0.12} & \multirow{2}{*}{ UD } & Open & 5.79 & 5.91 \\
\hline & & Close & 5.62 & 5.70 \\
\hline \multirow{2}{*}{0.17} & \multirow{2}{*}{ UD } & Open & 6.21 & 6.32 \\
\hline & & Close & 6.03 & 6.14 \\
\hline \multirow{2}{*}{0.28} & \multirow{2}{*}{ UD } & Open & 6.77 & 6.86 \\
\hline & & Close & 6.54 & 6.71 \\
\hline
\end{tabular}
$x_{1}=0.4, y_{1}=0.4, h=0.05, h_{p}=0.1 \mathrm{~h}$.

is clear that among the three cases of the boundary conditions, C-C shells have higher natural frequencies than those with C-S and S-S boundary conditions. Furthermore, Tables 13-16 show that by changing the 
Table 9. Fundamental frequencies of the spherical shell. Geometrical parameters are: $\varphi_{0}=\frac{\pi}{8}, \varphi_{1}=\frac{\pi}{2}, h=0.05 \mathrm{~m}$, $R=1 \mathrm{~m}, h_{p}=0.1 \mathrm{~h}, n=1$.

\begin{tabular}{cccccc}
\hline $\boldsymbol{V}_{\boldsymbol{C} N \boldsymbol{T}}^{*}$ & Type & Condition & C-C & C-S & S-S \\
\hline \multirow{2}{*}{0.12} & \multirow{2}{*}{ FGX } & Open & 709.45 & 689.76 & 682.59 \\
& & Close & 709.01 & 689.31 & 682.22 \\
0.17 & \multirow{2}{*}{ FGX } & Open & 791.89 & 766.53 & 756.08 \\
& & Close & 791.60 & 766.23 & 755.84 \\
0.28 & \multirow{2}{*}{ FGX } & Open & 880.71 & 846.03 & 830.13 \\
& & Close & 880.58 & 845.89 & 830.03 \\
0.12 & \multirow{2}{*}{ FGV } & Open & 695.31 & 685.59 & 650.60 \\
& & Close & 694.92 & 685.26 & 650.37 \\
0.17 & \multirow{2}{*}{ FGV } & Open & 771.04 & 757.38 & 713.12 \\
& & Close & 770.79 & 757.19 & 712.96 \\
0.28 & \multirow{2}{*}{ FGV } & Open & 846.99 & 824.41 & 771.87 \\
& & Close & 846.88 & 824.34 & 771.79 \\
0.12 & \multirow{2}{*}{ FGO } & Open & 688.79 & 6.88 .19 & 657.11 \\
& & Close & 688.39 & 672.78 & 656.81 \\
0.17 & \multirow{2}{*}{ FGO } & Open & 761.90 & 741.61 & 720.18 \\
& & Close & 761.65 & 741.34 & 719.99 \\
& \multirow{2}{*}{ FGO } & Open & 8.33 & 804.70 & 776.16 \\
& & Close & 833.11 & 804.58 & 776.07 \\
\hline
\end{tabular}

Table 10. Fundamental frequencies of toro circular shell. Geometrical parameters are: $\varphi_{0}=\frac{\pi}{3}, \varphi_{1}=\frac{2 \pi}{3}, h=0.2 \mathrm{~m}$, $h_{p}=0.1 \mathrm{~h}, R_{b}=1.5 \mathrm{~m}, R=3 \mathrm{~m}$.

\begin{tabular}{|c|c|c|c|c|c|}
\hline$V_{C N T}^{*}$ & Type & Condition & $\mathrm{C}-\mathrm{C}$ & $\mathrm{C}-\mathrm{S}$ & S-S \\
\hline \multirow{2}{*}{0.12} & \multirow{2}{*}{ FGX } & Open & 254.63 & 252.41 & 251.21 \\
\hline & & Close & 254.57 & 252.33 & 251.12 \\
\hline \multirow{2}{*}{0.17} & \multirow{2}{*}{ FGX } & Open & 282.18 & 280.13 & 278.91 \\
\hline & & Close & 282.15 & 280.08 & 278.85 \\
\hline \multirow{2}{*}{0.28} & \multirow{2}{*}{ FGX } & Open & 318.90 & 317.17 & 316.07 \\
\hline & & Close & 318.88 & 317.15 & 316.04 \\
\hline \multirow{2}{*}{0.12} & \multirow{2}{*}{ FGV } & Open & 251.64 & 251.60 & 251.59 \\
\hline & & Close & 251.55 & 251.51 & 251.50 \\
\hline \multirow{2}{*}{0.17} & \multirow{2}{*}{ FGV } & Open & 278.77 & 278.47 & 278.42 \\
\hline & & Close & 278.71 & 278.41 & 278.35 \\
\hline \multirow{2}{*}{0.28} & \multirow{2}{*}{ FGV } & Open & 314.97 & 313.76 & 313.53 \\
\hline & & Close & 314.93 & 313.72 & 313.48 \\
\hline \multirow{2}{*}{0.12} & \multirow{2}{*}{ FGO } & Open & 250.13 & 249.05 & 248.80 \\
\hline & & Close & 250.05 & 248.96 & 248.71 \\
\hline \multirow{2}{*}{0.17} & \multirow{2}{*}{$\mathrm{FGO}$} & Open & 277.05 & 276.20 & 275.96 \\
\hline & & Close & 277.05 & 276.15 & 275.90 \\
\hline \multirow{2}{*}{0.28} & \multirow{2}{*}{$\mathrm{FGO}$} & Open & 312.97 & 312.36 & 312.21 \\
\hline & & Close & 312.94 & 312.33 & 312.18 \\
\hline
\end{tabular}

Table 11. Fundamental frequencies of cycloidal shell. Geometrical parameters are: $\varphi=\frac{\pi}{12}, \varphi=\frac{5 \pi}{12}, h=0.1 \mathrm{~m}$, $h_{p}=0.1 \mathrm{~h}, r_{c}=1 \mathrm{~m}, R_{b}=2 \mathrm{~m}$.

\begin{tabular}{|c|c|c|c|c|c|}
\hline$V_{C N T}^{*}$ & Type & Condition & $\mathrm{C}-\mathrm{C}$ & C-S & S-S \\
\hline \multirow{2}{*}{0.12} & \multirow{2}{*}{ FGX } & Open & 261.36 & 242.99 & 214.83 \\
\hline & & Close & 261.14 & 242.88 & 214.70 \\
\hline \multirow{2}{*}{0.17} & \multirow{2}{*}{ FGX } & Open & 297.95 & 278.23 & 248.40 \\
\hline & & Close & 297.79 & 278.15 & 248.30 \\
\hline \multirow{2}{*}{0.28} & \multirow{2}{*}{ FGX } & Open & 340.85 & 317.08 & 286.92 \\
\hline & & Close & 340.79 & 317.02 & 286.86 \\
\hline \multirow{2}{*}{0.12} & \multirow{2}{*}{ FGV } & Open & 236.75 & 213.09 & 190.21 \\
\hline & & Close & 236.67 & 213.04 & 190.19 \\
\hline \multirow{2}{*}{0.17} & \multirow{2}{*}{ FGV } & Open & 268.04 & 242.84 & 218.54 \\
\hline & & Close & 267.98 & 242.80 & 218.53 \\
\hline \multirow{2}{*}{0.28} & \multirow{2}{*}{ FGV } & Open & 302.53 & 276.82 & 249.91 \\
\hline & & Close & 302.50 & 276.79 & 249.90 \\
\hline \multirow{2}{*}{0.12} & \multirow{2}{*}{ FGO } & Open & 229.57 & 209.49 & 186.78 \\
\hline & & Close & 229.43 & 209.35 & 186.63 \\
\hline \multirow{2}{*}{0.17} & \multirow{2}{*}{ FGO } & Open & 259.30 & 238.26 & 214.27 \\
\hline & & Close & 259.20 & 238.15 & 214.15 \\
\hline \multirow{2}{*}{0.28} & \multirow{2}{*}{$\mathrm{FGO}$} & Open & 291.7 & 270.07 & 243.96 \\
\hline & & Close & 291.64 & 269.99 & 243.58 \\
\hline
\end{tabular}

Table 12. Fundamental frequencies of elliptical shell. Geometrical parameters are: $\varphi_{0}=\frac{\pi}{6}, \varphi_{1}=\frac{5 \pi}{6}, h=0.2 \mathrm{~m}$, $a=3 \mathrm{~m}, b=2 \mathrm{~m}, R_{b}=0 \mathrm{~m}, h p=0.1 \mathrm{~h}, n=1$.

\begin{tabular}{|c|c|c|c|c|c|}
\hline$V_{C N T}^{*}$ & Type & Condition & $\mathrm{C}-\mathrm{C}$ & $\mathrm{C}-\mathrm{S}$ & S-S \\
\hline \multirow{2}{*}{0.12} & \multirow{2}{*}{ FGX } & Open & 231.68 & 216.74 & 202.51 \\
\hline & & Close & 231.64 & 216.69 & 202.46 \\
\hline \multirow{2}{*}{0.17} & \multirow{2}{*}{ FGX } & Open & 253.77 & 239.11 & 224.93 \\
\hline & & Close & 253.75 & 239.08 & 224.89 \\
\hline \multirow{2}{*}{0.28} & \multirow{2}{*}{ FGX } & Open & 271.42 & 258.49 & 245.69 \\
\hline & & Close & 271.41 & 258.47 & 245.68 \\
\hline \multirow{2}{*}{0.12} & \multirow{2}{*}{ FGV } & Open & 219.33 & 200.40 & 183.11 \\
\hline & & Close & 219.22 & 200.38 & 183.09 \\
\hline \multirow{2}{*}{0.17} & \multirow{2}{*}{ FGV } & Open & 239.86 & 220.52 & 202.49 \\
\hline & & Close & 239.85 & 220.51 & 202.48 \\
\hline \multirow{2}{*}{0.28} & \multirow{2}{*}{ FGV } & Open & 257.19 & 238.64 & 220.77 \\
\hline & & Close & 257.18 & 238.63 & 220.76 \\
\hline \multirow{2}{*}{0.12} & \multirow{2}{*}{ FGO } & Open & 216.10 & 201.20 & 187.53 \\
\hline & & Close & 216.04 & 201.13 & 187.47 \\
\hline \multirow{2}{*}{0.17} & \multirow{2}{*}{$\mathrm{FGO}$} & Open & 236.17 & 220.95 & 206.81 \\
\hline & & Close & 236.13 & 220.91 & 206.77 \\
\hline \multirow{2}{*}{0.28} & \multirow{2}{*}{$\mathrm{FGO}$} & Open & 253.11 & 238.16 & 223.98 \\
\hline & & Close & 253.09 & 238.14 & 223.96 \\
\hline
\end{tabular}


Table 13. Fundamental frequency of spherical panel $\varphi_{0}=\frac{\pi}{8}, \varphi_{1}=\frac{\pi}{2}, \vartheta=\frac{2 \pi}{3}, h=0.05 \mathrm{~m}, R=1 \mathrm{~m}, h p=0.1$ h.

\begin{tabular}{|c|c|c|c|c|}
\hline$V_{C N T}^{*}$ & Type & Condition & C-C-C-C & C-S-C-S \\
\hline \multirow{2}{*}{0.12} & \multirow{2}{*}{ FGX } & Open & 811.12 & 784.00 \\
\hline & & Close & 810.55 & 783.41 \\
\hline \multirow{2}{*}{0.17} & \multirow{2}{*}{ FGX } & Open & 901.86 & 871.27 \\
\hline & & Close & 901.51 & 870.94 \\
\hline \multirow{2}{*}{0.28} & \multirow{2}{*}{ FGX } & Open & 996.07 & 958.97 \\
\hline & & Close & 995.94 & 958.85 \\
\hline \multirow{2}{*}{0.12} & \multirow{2}{*}{ FGV } & Open & 777.66 & 758.08 \\
\hline & & Close & 777.20 & 757.60 \\
\hline \multirow{2}{*}{0.17} & \multirow{2}{*}{ FGV } & Open & 852.34 & 831.81 \\
\hline & & Close & 852.04 & 831.54 \\
\hline \multirow{2}{*}{0.28} & \multirow{2}{*}{ FGV } & Open & 923.00 & 897.71 \\
\hline & & Close & 922.82 & 897.55 \\
\hline \multirow{2}{*}{0.12} & \multirow{2}{*}{ FGO } & Open & 755.56 & 737.08 \\
\hline & & Close & 755.14 & 736.68 \\
\hline \multirow{2}{*}{0.17} & \multirow{2}{*}{ FGO } & Open & 821.97 & 800.44 \\
\hline & & Close & 821.76 & 800.25 \\
\hline \multirow{2}{*}{0.28} & \multirow{2}{*}{ FGO } & Open & 885.10 & 857.49 \\
\hline & & Close & 885.03 & 857.42 \\
\hline
\end{tabular}

Table 14. Fundamental frequencies of the toro circular panel. Geometrical parameters are: $\varphi_{0}=\frac{\pi}{3}, \varphi_{1}=\frac{2 \pi}{3}$, $\vartheta=\frac{2 \pi}{3}, h=0.2 \mathrm{~m}, R=3 \mathrm{~m}, R_{b}=1.5 \mathrm{~m}, h_{p}=0.1 \mathrm{~h}$.

\begin{tabular}{|c|c|c|c|c|}
\hline$V_{C N T}^{*}$ & Type & Condition & C-C-C-C & C-S-C-S \\
\hline \multirow{2}{*}{0.12} & \multirow{2}{*}{ FGX } & Open & 286.34 & 282.31 \\
\hline & & Close & 286.13 & 282.11 \\
\hline \multirow{2}{*}{0.17} & \multirow{2}{*}{ FGX } & Open & 325.13 & 320.35 \\
\hline & & Close & 324.97 & 320.20 \\
\hline \multirow{2}{*}{0.28} & \multirow{2}{*}{ FGX } & Open & 378.00 & 374.78 \\
\hline & & Close & 377.98 & 374.69 \\
\hline \multirow{2}{*}{0.12} & \multirow{2}{*}{ FGV } & Open & 279.06 & 273.77 \\
\hline & & Close & 278.83 & 273.57 \\
\hline \multirow{2}{*}{0.17} & \multirow{2}{*}{ FGV } & Open & 316.16 & 309.02 \\
\hline & & Close & 315.99 & 308.87 \\
\hline \multirow{2}{*}{0.28} & \multirow{2}{*}{ FGV } & Open & 351.02 & 349.00 \\
\hline & & Close & 350.98 & 348.96 \\
\hline \multirow{2}{*}{0.12} & \multirow{2}{*}{$\mathrm{FGO}$} & Open & 276.56 & 273.44 \\
\hline & & Close & 276.33 & 273.22 \\
\hline \multirow{2}{*}{0.17} & \multirow{2}{*}{ FGO } & Open & 313.27 & 309.50 \\
\hline & & Close & 313.10 & 309.34 \\
\hline \multirow{2}{*}{0.28} & \multirow{2}{*}{$\mathrm{FGO}$} & Open & 338.65 & 336.17 \\
\hline & & Close & 338.64 & 336.15 \\
\hline
\end{tabular}

Table 15. Fundamental frequency of the cycloidal panel. Geometrical parameters are: $\varphi_{0}=\frac{\pi}{12}, \varphi_{1}=\frac{5 \pi}{12}, \vartheta=\frac{2 \pi}{3}$, $h=0.1 \mathrm{~m}, R_{b}=2 \mathrm{~m}, r_{c}=1 \mathrm{~m}, h_{p}=0.1 \mathrm{~h}$.

\begin{tabular}{|c|c|c|c|c|}
\hline$V_{C N T}^{*}$ & Type & Condition & C-C-C-C & C-S-C-S \\
\hline \multirow{2}{*}{0.12} & \multirow{2}{*}{ FGX } & Open & 260.68 & 260.07 \\
\hline & & Close & 260.51 & 259.90 \\
\hline \multirow{2}{*}{0.17} & \multirow{2}{*}{ FGX } & Open & 294.51 & 294.15 \\
\hline & & Close & 294.42 & 294.05 \\
\hline \multirow{2}{*}{0.28} & \multirow{2}{*}{ FGX } & Open & 330.13 & 328.82 \\
\hline & & Close & 330.10 & 328.80 \\
\hline \multirow{2}{*}{0.12} & \multirow{2}{*}{ FGV } & Open & 235.61 & 235.45 \\
\hline & & Close & 235.52 & 235.37 \\
\hline \multirow{2}{*}{0.17} & \multirow{2}{*}{ FGV } & Open & 262.57 & 262.38 \\
\hline & & Close & 262.52 & 262.32 \\
\hline \multirow{2}{*}{0.28} & \multirow{2}{*}{$\mathrm{FGV}$} & Open & 290.50 & 289.08 \\
\hline & & Close & 290.46 & 289.04 \\
\hline \multirow{2}{*}{0.12} & \multirow{2}{*}{ FGO } & Open & 225.62 & 225.49 \\
\hline & & Close & 225.54 & 225.41 \\
\hline \multirow{2}{*}{0.17} & \multirow{2}{*}{ FGO } & Open & 250.32 & 249.09 \\
\hline & & Close & 250.27 & 249.06 \\
\hline \multirow{2}{*}{0.28} & \multirow{2}{*}{ FGO } & Open & 274.39 & 272.87 \\
\hline & & Close & 274.38 & 272.86 \\
\hline
\end{tabular}

Table 16. Fundamental frequency of the elliptical panel. Geometrical parameters are: $\varphi_{0}=\frac{\pi}{6}, \varphi_{1}=\frac{5 \pi}{6}, \vartheta=\frac{2 \pi}{3}$, $h=0.2 \mathrm{~m}, R_{b}=0, h_{p}=0.1 \mathrm{~h}, a=3, b=2$.

\begin{tabular}{|c|c|c|c|c|}
\hline$V_{C N T}^{*}$ & Type & Condition & C-C-C-C & C-S-C-S \\
\hline \multirow{2}{*}{0.12} & \multirow{2}{*}{ FGX } & Open & 257.83 & 257.38 \\
\hline & & Close & 257.72 & 257.26 \\
\hline \multirow{2}{*}{0.17} & \multirow{2}{*}{ FGX } & Open & 289.32 & 278.35 \\
\hline & & Close & 289.23 & 278.33 \\
\hline \multirow{2}{*}{0.28} & \multirow{2}{*}{ FGX } & Open & 307.81 & 295.62 \\
\hline & & Close & 307.80 & 295.61 \\
\hline \multirow{2}{*}{0.12} & \multirow{2}{*}{ FGV } & Open & 250.54 & 250.32 \\
\hline & & Close & 250.48 & 250.26 \\
\hline \multirow{2}{*}{0.17} & \multirow{2}{*}{ FGV } & Open & 272.96 & 265.89 \\
\hline & & Close & 272.94 & 265.87 \\
\hline \multirow{2}{*}{0.28} & \multirow{2}{*}{ FGV } & Open & 288.04 & 279.80 \\
\hline & & Close & 288.02 & 279.78 \\
\hline \multirow{2}{*}{0.12} & \multirow{2}{*}{$\mathrm{FGO}$} & Open & 241.96 & 239.20 \\
\hline & & Close & 241.83 & 239.17 \\
\hline \multirow{2}{*}{0.17} & \multirow{2}{*}{ FGO } & Open & 263.69 & 255.56 \\
\hline & & Close & 263.67 & 255.54 \\
\hline \multirow{2}{*}{0.28} & \multirow{2}{*}{ FGO } & Open & 278.23 & 268.94 \\
\hline & & Close & 278.22 & 268.93 \\
\hline
\end{tabular}




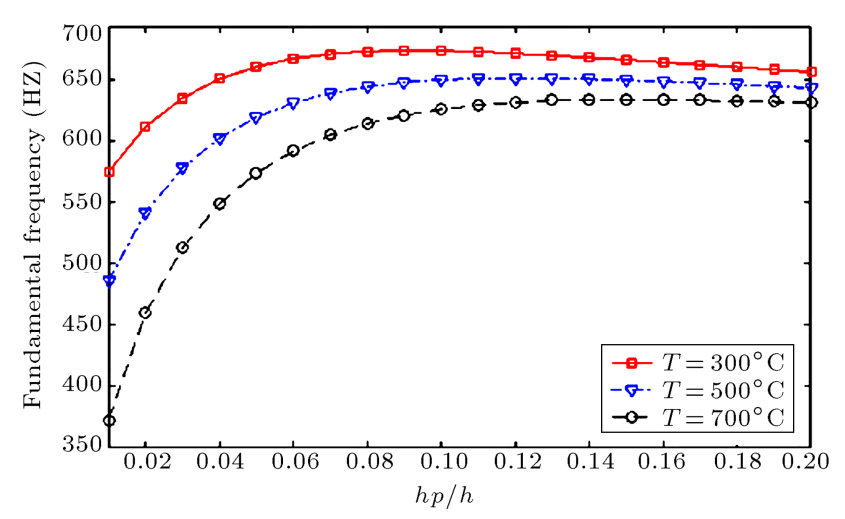

Figure 7. Fundamental frequency of the spherical shell with ratio of thickness of piezoelectric layer to core layer (C-S).

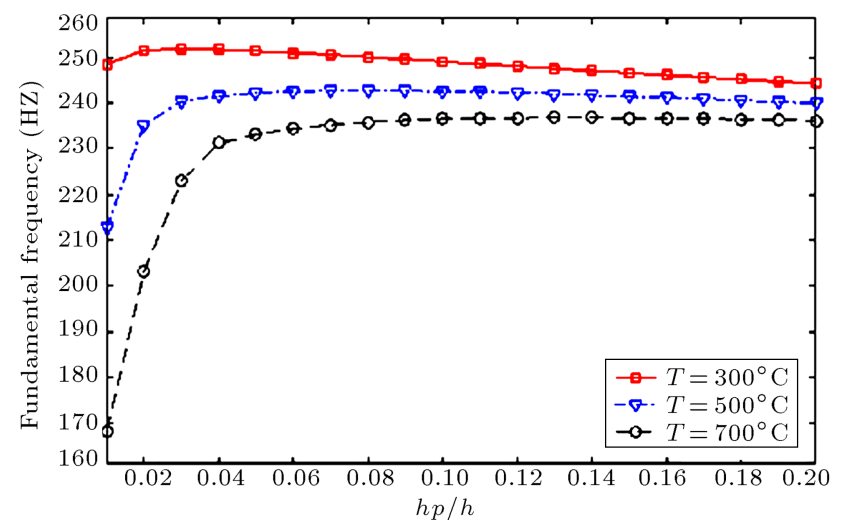

Figure 8. Fundamental frequency of the toro circular shell with ratio of thickness of piezoelectric layer to core layer (C-S).

electrical state to open circuit, fundamental frequencies of the panels rise. All of the case studies show that as the CNT volume fraction increases, the fundamental frequencies increase. Furthermore, among the four possible graded patterns of the CNTs, the FG-X type of CNT distribution produces higher frequencies and the lowest frequencies are relevant to the FG-O pattern. As expected, among the two boundary conditions, CCCC panels have higher natural frequencies than the CSCS panels. Figures 7-14 show the fundamental frequencies of structures in a thermal environment. To investigate the thermal effect, the distribution function of CNT is assumed based on FGO pattern and the volume fraction is 0.12 . According to these figures, the fundamental frequencies are reduced at higher temperatures. Thus, by increasing the ratio of the piezoelectric layer thickness to the core thickness, the effect of temperature is negligible and can be ignored. Thus, the difference between fundamental frequencies at different temperatures is reduced.

\section{Conclusion}

In this study, the General Differential Quadrature

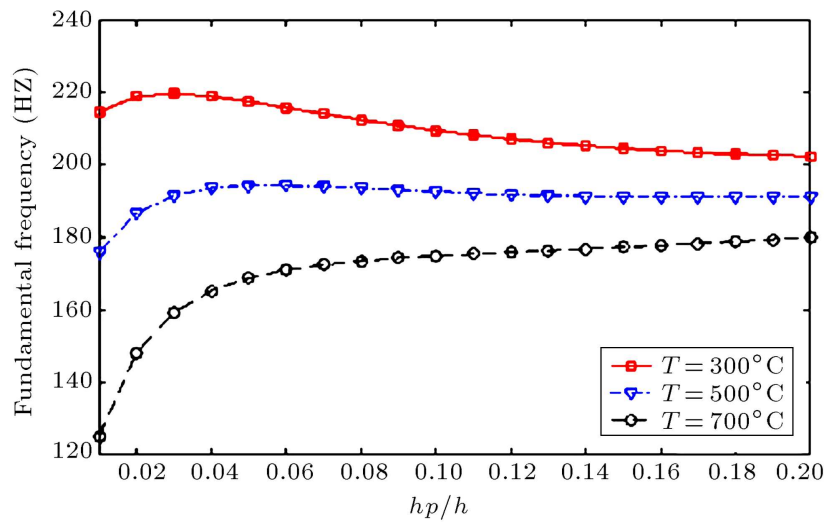

Figure 9. Fundamental frequency of cycloidal shell with the ratio of thickness of piezoelectric layer to core layer (C-S).

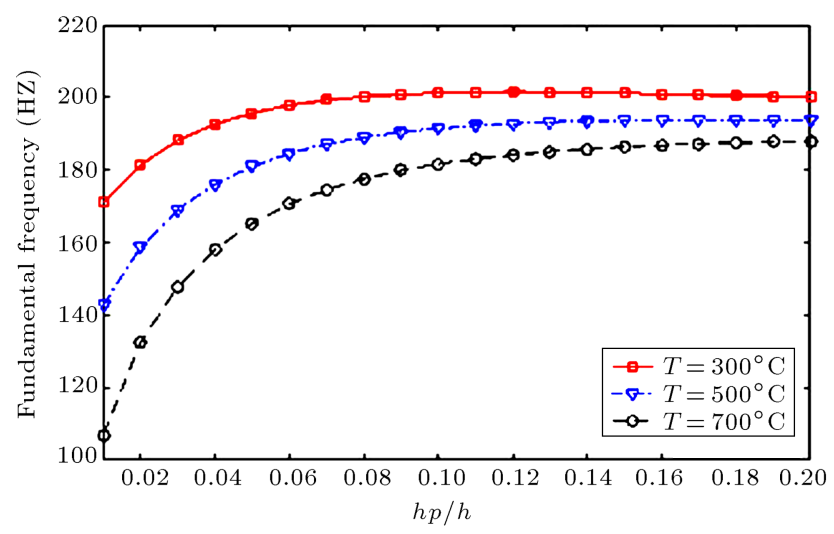

Figure 10. Fundamental frequency of elliptical shell with the ratio of thickness of piezoelectric layer to core layer (C-S).

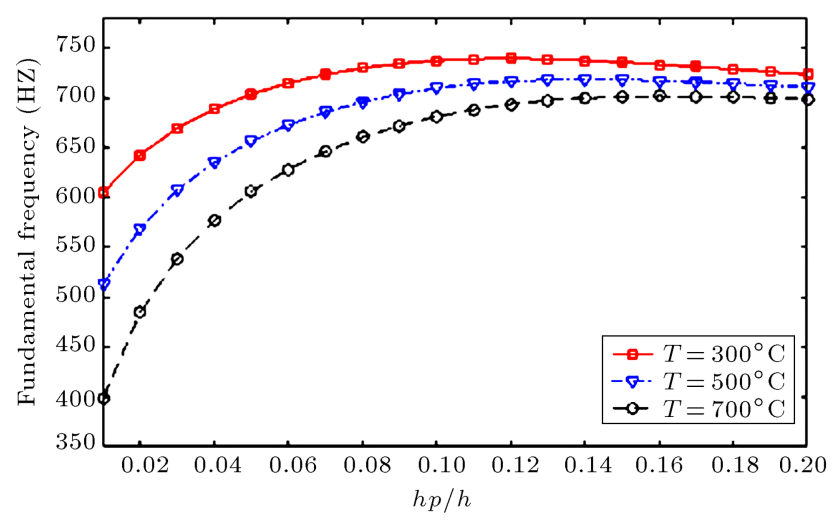

Figure 11. Fundamental frequency of the spherical panel with ratio of thickness of piezoelectric layer to core layer (C-S).

(GDQ) method was implemented to examine the free vibration of double curved structures. The core of the structures is made of Carbon Nanotubes (CNTs). The mechanical properties were obtained based on the modified rule of mixture. The upper and lower surfaces were covered with piezoelectric layers. The 


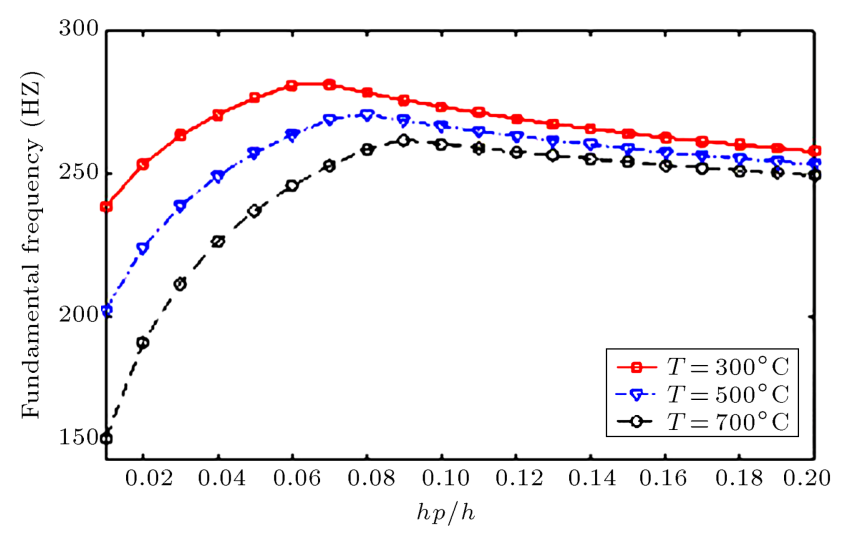

Figure 12. Fundamental frequency of the toro circular panel with ratio of thickness of piezoelectric layer to core layer (C-S).

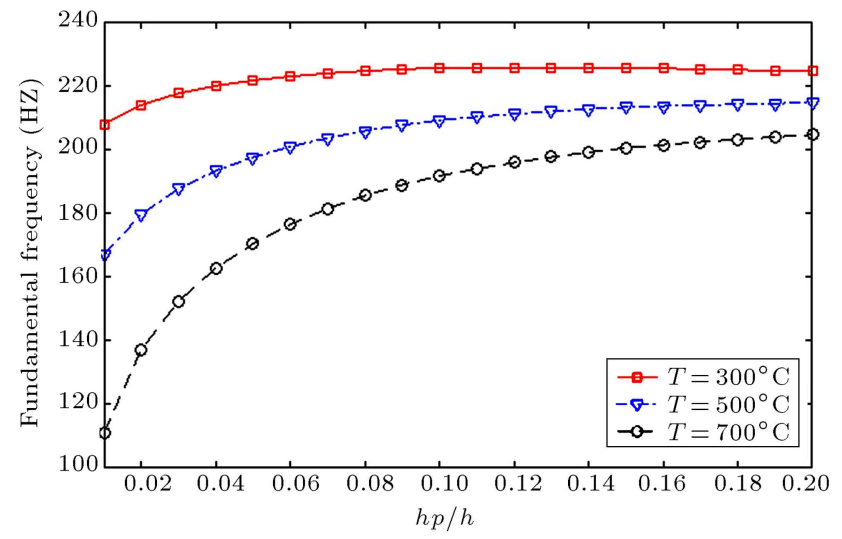

Figure 13. Fundamental frequency of the cycloidal panel with ratio of thickness of piezoelectric layer to core layer (C-S).

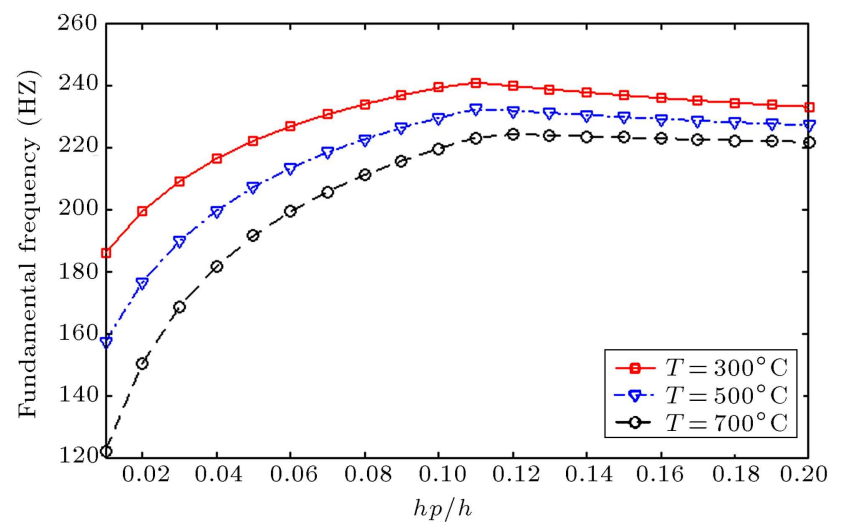

Figure 14. Fundamental frequency of the elliptical panel with ratio of thickness of piezoelectric layer to core layer (C-S).

numerical results are employed to examine the effects of the boundary conditions, volume fractions, electrical condition, thickness of piezoelectric layers, and kinds of structures. It was observed that the frequency of the whole structures rose when the FGX pattern was used. In addition, the frequency rose when the volume frac-

tion increased. The open circuit condition has a higher frequency than the closed circuit. In the high mode number, the difference between open circuit and closed circuit frequencies widens. The effect of the mechanical boundary conditions on the fundamental frequencies is studied; accordingly, the clamped-clamped boundary condition has a higher frequency than others. The effect of a thermal environment is investigated. Due to the increase in the environment temperature, the frequency parameter tends to decrease. By increasing the thickness of piezoelectric layers, the effect of the thermal environment is reduced.

\section{Nomenclature}

$\alpha$

$z$

$z_{c}$

$h$

$h_{p}$

$R_{b}$

$a$

$b$

$R_{\alpha}$

$R_{\beta}$

$R_{0}$

$V_{\text {tot }}$

$r_{c}$

$U$

$V$

W

$u$

$v$

$w$

$\Psi_{\alpha}$

$\Psi_{\beta}$

$A_{i}$

$N$

$A_{i j}$

$B_{i j}$

$D_{i}$

$P_{i j}$
Value of the meridional direction

Value of the circumferential direction Value of the thickness direction

Value of the piezoelectric thickness direction

Thickness of the structures

Thickness of piezoelectric

Distance between geometric axis and revolution axis

Semi major axis

Semi minor axis

Radius in the meridional direction

Radius in the circumferential direction

Horizontal radius

Potential energy

Radius of the produced circle of cycloid curve

Displacement in the meridional

direction

Displacement in the circumferential direction

Displacement in the thickness direction

Displacement of reference plane in the meridional direction

Displacement of reference plane in the circumferential direction

Displacement of reference plane in the thickness direction

Rotation around $\alpha$ axis

Rotation around $\beta$ axis

Lamé parameters

Resultant force

Extensional stiffness

Bending-extensional coupling

stiffnesses

Electrical displacements

Pyroelectric property 


$\begin{array}{ll}e_{i j} & \text { Piezoelectric stress constant } \\ p & \text { Piezoelectric } \\ c & \text { Core } \\ \alpha_{i j} & \text { Thermal expansion } \\ \Delta T & \text { Temperature gradient } \\ V^{*} & \text { Total volume fraction } \\ \eta_{i} & \text { Efficiency parameter } \\ \nu & \text { Poisson's coefficient } \\ \rho & \text { Density } \\ E_{i j} & \text { Young modulus } \\ U_{t o t} & \text { Strain energy } \\ K_{t o t} & \text { Kinetic energy } \\ I_{i} & \text { Moment of inertia } \\ C_{i j}, C_{p i j} & \text { Weighting coefficients } \\ M(\alpha) & \text { Legendre polynomial } \\ {[M]} & \text { Mass matrix } \\ {[K]} & \text { Stiffness matrix } \\ \chi & \text { Curvature } \\ M & \text { Moment resultant } \\ D_{i j} & \text { Bending stiffness } \\ T & \text { Thermal } \\ k_{i j} & \text { Dielectric permittivity } \\ \phi & \text { The electric potential }\end{array}$

\section{References}

1. Qatu, M., Sullivan, R.W., and Wanga, W. "Recent research advances on the dynamic analysis of composite shells 2000-2009", Compos. Struct., 93, pp. 14-31 (2010).

2. Alijani, F. and Amabili, M. "Non-linear vibrations of shells: A literature review from 2003 to 2013", Int. J. Nonlin Mech., 58, pp. 233-257 (2014).

3. Kulkarni, H., Khusru, K.Z., and Shravan Aiyappa, K. "Application of piezoelectric technology in automotive systems", Mater Today Proc., 5, pp. 21299-21304 (2018).

4. Tornabene, F. "2-D GDQ solution for free vibrations of anisotropic doubly-curved shells and panels of revolution", Compos. Struct., 93, pp. 1854-1876 (2011).

5. Viola, E., Tornabene, F., and Fantuzzi, N. "Static analysis of completely doubly-curved laminated shells and panels using general higher-order shear deformation theories", Compose. Struc., 101, pp. 59-93 (2013).

6. Shooshtari, A. and Razavi, S. "Large-amplitude free vibration of magneto-electro-elastic curved panels", Sci. Iran., 23, pp. 2606-2615 (2016).

7. Pang, F., Li, H., Cui, J., Du, Y., and Gao, C. "Application of flügge thin shell theory to the solution of free vibration behaviors for spherical-cylindricalspherical shell: A unified formulation", Eur. J. Mech A-Solid, 74, pp. 381-393 (2019).
8. Rout, M., Hota, S., and Karmakar, A. "Thermoelastic free vibration response of graphene reinforced laminated composite shells", Eng. Struct, 178, pp. 179190 (2019).

9. Pang, F., Li, H., Wang, X., Miao, X., and Li, S. "A semi analytical method for the free vibration of doublycurved shells of revolution", Comput. Math Appl., 75, pp. 3249-3268 (2018).

10. Awrejcewicz, J., Kurpa, L., and Shmatko, T. "Linear and nonlinear free vibration analysis of laminated functionally graded shallow shells with complex plan form and different boundary conditions", Int. J. Nonlin Mech., 107, pp. 161-169 (2018).

11. Fang, X., Zhu, C., Liu, J., and Liu, X. "Surface energy effect on free vibration of nano-sized piezoelectric double-shell structures", Physica B Condens Matter., 529, pp. 41-56 (2018).

12. Zhoua, Z., Nia, Y., Zhua, S., Tonga, Z., Sunb, J., and $\mathrm{Xua}, \mathrm{X}$. "An accurate and straightforward approach to thermo-electro-mechanical vibration of piezoelectric fiber-reinforced composite cylindrical shells", Compose. Struc., 207, pp. 292-303 (2019).

13. Mallek, H., Jrad, H., Wali, M., and Dammak, F. "Piezoelastic response of smart functionally graded structure with integrated piezoelectric layers using discrete double directors shell element", Compose. Struc., 210, pp. 354-366 (2019).

14. Akbari Alashti, A., Khorsand, M., and Tarahhomi, M.H. "Thermo-elastic analysis of a functionally graded spherical shell with piezoelectric layers by differential quadrature method", Sci. Iran., 20, pp. 109-119 (2013).

15. Behjat, B., Salehi, M., Armin, A., Sadighi, M., Abbasi, M. "Static and dynamic analysis of functionally graded piezoelectric plates under mechanical and electrical loading", Sci. Iran., 20, pp. 986-994 (2011).

16. Wang, Q., Shi, Q., Liang, D., and Pang, F. "Free vibration of four-parameter functionally graded moderately thick doubly-curved panels and shells of revolution with general boundary conditions", Appl.Math. Model, 4, pp. 705-734 (2017).

17. Reddy, I.N., Mechanics of Laminated Composite Plates and Shells, Washington, D.C, CRC Press (2004).

18. Ventsel, E. and Kravthammer, T. "Thin plates and shells", New York, NY10016 (2001).

19. Sayyaadi, H. and Askari Farsangi, M.A. "An analytical solution for dynamic behavior of thick doubly curved functionally graded smart panels", Compose. Struct., 107, pp. 88-102 (2014).

20. Kiani, Y. "Free vibration of functionally graded carbon nanotube reinforced composite plates integrated with piezoelectric layers", Comput. Math Appl., 72, pp. 2433-2449 (2016).

21. Jafari, A.A., Khalili, S.M.R., and Tavakolian, M. "Nonlinear vibration of functionally graded cylindrical shells embedded with a piezoelectric layer", Thin Walled Struct., 79, pp. 8-15 (2014). 
22. Song, Z.G., Zhang, L.W., and Liew, K.M. "Vibration analysis of CNT-reinforced functionally graded composite cylindrical shells in thermal environments", Int. J. Mech Sci., 115-116, pp. 339-347 (2016).

23. Zghal, S., Farikha, A., and dammak, F. "Free vibration analysis of carbon nanotube-reinforced functionally graded composite shell structures", Appl. Math. Model, 53, pp. 132-155 (2018).

24. Arshid, E. and Khorshidvand, A.R. "Free vibration analysis of saturated porous FG circular plates integrated with piezoelectric actuators via differential quadrature method", Thin-Walled Struct, 125, pp. 220-233 (2018).

25. Bodaghi, M. and Shakeri, M. "An analytical approach for free vibration and transient response of functionally graded piezoelectric cylindrical panels subjected to impulsive loads". Compose. Struct., 94, pp. 1721-1735 (2012).

26. Shu, C. "Free vibration analysis of composite laminated conical shells by generalized differential quadrature", J. Sound Vib., 194, pp. 587-604 (1996).

27. Behjat, B., Salehi, M., Sadighi, M., Armin, A., and Abbasi, M. "Static, dynamic, and free vibration analysis of functionally graded piezoelectric panels using finite element method", J. Intell Mater Syst. Struct., 20, pp. 1635-1646 (2009).

28. Ansari, R., Torabi, J., and Faghih Shojaei, M. "Vibrational analysis of functionally graded carbon nanotube reinforced composite", Eur. J. Mech A-Solid, 60, pp. 166-182 (2016).

29. Xie, X., Zheng, H., and Jin, G. "Free vibration of four-parameter functionally graded spherical and parabolic shells of revolution with arbitrary boundary conditions", Compos Part B: Eng., 77, pp. 59-73 (2015).

30. Zhang, H., Shi, D., Wang, Q., and Qin, B. "Free vibration of functionally graded parabolic and circular panels with general boundary conditions", Curved and Layer. Struct., 4, pp. 52-84 (2017).

31. Shen, H.S. and Xiang, Y. "Nonlinear analysis of nanotube-reinforced composite beams resting on elastic foundations in thermal environments", Eng. Struct., 56, pp. 698-708 (2013).

32. Han, Y. and Elliott, J. "Molecular dynamics simulations of the elastic properties of polymer/carbon nanotube composites", Comput. Mater. Sci, 39, pp. 315-323 (2007).

33. Shariyat, M. "Dynamic buckling of imperfect laminated plates with piezoelectric sensors and actuators subjected to thermo-electro-mechanical loadings, considering the temperature-dependency of the material properties", Compose. Struct., 88, pp. 228-239 (2016).

\section{Biographies}

Siros Khorshidi graduated in Mechanical Engineering from Tehran Polytechnic in 2016. He also received his BSc in Material Science and Engineering from the University of Tehran in 2012. His current research interests include nonlinear vibration.

Saeed Saber-Samandari is currently an Assistant Professor at New Technologies Research Center of the Amirkabir University of Technology. He has studied experimental, analytical, and computational aspects of the properties of engineered materials, particularly on the sub-microscopic and nanoscopic length scales.

Manouchehr Salehi obtained his $\mathrm{PhD}$ in Mechanical En gineering from Lancaster University. He is currently a Professor at the Department of Mechanical Engineering at the Amirkabir University of Technology. His current research contains composites, plates and shells, elasticity, viscoelasticity, and nanomechanics. 